TRANSACTIONS OF THE

AMERICAN MATHEMATICAL SOCIETY

Volume 363, Number 11, November 2011, Pages 5971-6005

S 0002-9947(2011)05298-0

Article electronically published on May 2, 2011

\title{
REFLECTION SUBGROUPS OF FINITE AND AFFINE WEYL GROUPS
}

\author{
M. J. DYER AND G. I. LEHRER
}

\begin{abstract}
We give complete classifications of the reflection subgroups of finite and affine Weyl groups from the point of view of their root systems. A short case-free proof is given of the well-known classification of the isomorphism classes of reflection subgroups using completed Dynkin diagrams, for which there seems to be no convenient source in the literature. This is used as a basis for treating the affine case, where we give two distinct 'on the nose' classifications of reflection subgroups, as well as reproving Coxeter's conjecture on the isomorphism classes of reflection groups which occur. Geometric and combinatorial aspects of root systems play an essential role. Parameter sets for various types of subsets of roots are interpreted in terms of alcove geometry and the Tits cone, and combinatorial identities are derived.
\end{abstract}

\section{INTRODUCTION}

The classification of the reflection subgroups of Coxeter groups is a fundamental issue, arising in many mathematical contexts. Examples include Carter's determination of the conjugacy classes of the finite Weyl groups, the determination of the action of a crystallographic finite Coxeter group on the cohomology of its associated toric variety, and in the study of root subgroups of reductive groups (see below for more details). In this work we give complete case-free classifications of the reflection subgroups of crystallographic Weyl groups of finite and affine types. Group theoretic and combinatorial applications are given in $\$ \sqrt{6.15}$ and 6.16. In a sequel 7 to this work, we explore these ideas in the context of loop Kac-Moody Lie algebras.

1.1. Let $V$ be a Euclidean space, that is, a real vector space with a positive definite symmetric bilinear form which we write $\langle-,-\rangle: V \times V \rightarrow \mathbb{R}$. Let $\Phi$ be a finite reduced root system in $V$, in the sense of 2 . The following basic facts may be found in 2] or [27]. For $\alpha \in \Phi$, we have the corresponding coroot $\alpha^{\vee}=\frac{2 \alpha}{\langle\alpha, \alpha\rangle}$. The set $\Phi^{\vee}:=\left\{\alpha^{\vee} \mid \alpha \in \Phi\right\}$ is again a reduced root system in $V$, called the root system dual to $\Phi$.

For $\alpha \in \Phi$ the corresponding reflection $s_{\alpha}: V \rightarrow V$ is defined by

$$
s_{\alpha}(v)=v-\left\langle\alpha^{\vee}, v\right\rangle \alpha \quad(v \in V) .
$$

The Weyl group $W=W(\Phi)$ of $\Phi$ is the group generated by the reflections $s_{\alpha}$, $\alpha \in \Phi$, and it is well known that any reflection in $W$ is of the form $s_{\alpha}$ for some

Received by the editors December 13, 2009 and, in revised form, January 26, 2010.

2000 Mathematics Subject Classification. Primary 20F55, 51F15.

(C)2011 American Mathematical Society 
$\alpha \in \Phi$. In this work we shall assume that $W$ (or $\Phi$ ) is crystallographic; this means that $\left\langle\alpha, \beta^{\vee}\right\rangle \in \mathbb{Z}$ for all $\alpha, \beta \in \Phi$.

A subset $\Phi_{+} \subseteq \Phi$ is a positive system if it consists of the roots of $\Phi$ which are positive relative to some vector space total ordering of $V$. Such an ordering is defined by its cone $V_{+} \subseteq V$ of 'positive elements', which is closed under positive linear combinations, and satisfies $V=V_{+} \cup-V_{+} \cup\{0\}$ (a disjoint union). A subset $\Lambda \subseteq \Phi$ is a simple system if $\Lambda$ is linearly independent and every root of $\Phi$ is a linear combination of $\Lambda$ in which all non-zero coefficients have the same sign, i.e. are either all positive or all negative. It is well known that each positive system contains a unique simple system $\Lambda \subseteq \Phi_{+}$, and conversely. Moreover, the positive systems (and hence the simple systems) of $\Phi$ are in bijection with $W$, which acts on them regularly. That is, given two simple systems $\Lambda_{1}, \Lambda_{2}$ of $\Phi$, there is a unique element $w \in W$ such that $\Lambda_{2}=w \Lambda_{1}$.

1.2. A root subsystem of $\Phi$ is a subset $\Psi \subseteq \Phi$ which is itself a root system. This is equivalent to the requirement that for $\alpha, \beta \in \Psi, s_{\alpha}(\beta) \in \Psi$.

Positive subsystems and simple subsystems of $\Phi$ are respectively defined as the positive systems and simple systems of a root subsystem of $\Phi$.

A reflection subgroup $W^{\prime}$ of $W$ is a subgroup which is generated by a subset of the reflections $s_{\alpha}(\alpha \in \Phi)$. Any such subgroup has an associated set $\Psi=\Psi\left(W^{\prime}\right)$ of roots of $\Phi$, where $\Psi=\left\{\alpha \in \Phi \mid s_{\alpha} \in W^{\prime}\right\}$. Then $\Psi$ is clearly a root subsystem of $\Phi$, and the correspondence $W^{\prime} \leftrightarrow \Psi\left(W^{\prime}\right)$ is a bijection between reflection subgroups of $W$ and root subsystems of $\Phi$. In this work, we shall discuss the classification of root subsystems under various types of equivalence (isomorphism, $W$-conjugacy or equality) for both the finite cases and the associated affine root systems. Our treatment therefore includes a classification of reflection subgroups under the corresponding equivalence.

Definition 1. A root subsystem $\Psi \subseteq \Phi$ is closed if, whenever $\alpha, \beta \in \Psi$ and $\alpha+\beta \in \Phi$, then $\alpha+\beta \in \Psi$.

1.3. History of the finite case. In this subsection, we briefly review the history of the classification of reflection subgroups of finite Weyl groups. It is known that the root subsystem $\Psi$ is closed if and only if $\Psi=\mathbb{Z} \Psi \cap \Phi$ (see [13, §4]1]). Some sources (e.g. [12] and [13]) use the term 'root subsystem' to refer to what we here call a closed root subsystem.

Simple arguments reduce the problem of determining the closed root subsystems (up to isomorphism) to that of determining the maximal rank closed root subsystems. The closed root subsystems were determined up to isomorphism by Borel and de Siebenthal [1] (who treated the maximal rank ones) and by Dynkin [11, using the connection between crystallographic Weyl groups and Lie groups or Lie algebras. A treatment of these results directly in terms of root systems may be found in [21].

It is also well known that the classification of all subsystems up to isomorphism may be deduced from that of the closed subsystems. Carter [3] describes an algorithm for determining the isomorphism types of all root subsystems, similar to the algorithms from the results of Borel and de Siebenthal and of Dynkin for the closed subsystems. The paper [12] gives a case-by-case description of the reflection

\footnotetext{
${ }^{1}$ There is a simple proof of this and of the claim in op. cit., Lemma 4.1 using [21, Prop. 12.3]
} in place of the reference to 11 . 
subgroups of finite Coxeter groups up to isomorphism (even up to automorphisms of $W$ in the case of maximal reflection subgroups). In the case of Weyl groups, the results are based on the tables in Dynkin [11, Ch 2, §5], giving closed subsystems and maximal closed subsystems of $\Phi$ up to $W$-action. In the book [22] the classification of reflection subgroups up to isomorphism was extended from Weyl groups to all unitary (complex) reflection groups. The classifications in both [12] and [22] apply to the non-crystallographic finite Coxeter groups, as well as Weyl groups.

The algorithm producing the reflection subgroups of finite Weyl groups $W$ up to isomorphism as described in [3] is attributed by Carter to Borel and de Siebenthal [1, and it is used by Carter for the determination of the conjugacy classes of the Weyl group $W$. This classification also arises in the study of Weyl group actions on the cohomology of their corresponding toric varieties (see [28, 23]) and in several other contexts, such as the generation of subgroups of reductive groups by root subgroups [15]. It therefore seems desirable to have a clear, concise and selfcontained treatment of this result in the finite case, independent of the classification of finite Weyl groups. We give such an account in \$2. Our arguments are closely analogous to those of Dynkin, which we describe in an appendix, though we do not know of any simple and natural way to deduce the final results directly from those of either Dynkin or Borel and de Siebenthal.

1.4. The plan of this work. After treating the finite case in $₫ 2$, in $\oiint_{3}$ we provide background concerning affine Weyl groups. A classification (up to isomorphism) of the reflection subgroups of an affine Weyl group in terms of those of the corresponding finite Weyl group was conjectured and partly proved by Coxeter [5], and completed in [8]. The paper [12] includes explicit lists of the possible isomorphism types of maximal reflection subgroups and describes a procedure by which all reflection subgroups of a given affine Weyl group can be obtained. The latter gives a surjective map from implicitly defined combinatorial data to the set of reflection subgroups. That paper also includes classifications of maximal finite-index (indecomposable) reflection subgroups up to automorphisms and computations of their index, recorded in tables. A particularly striking result there (see Theorem 1 of op. cit and [13, Remark 5.1]) is that "most" maximal rank indecomposable reflection subgroups of indecomposable affine Weyl groups are determined, up to the action of automorphisms of the affine Weyl group, by their type and index.

In contrast, our main results in the affine setting provide two different bijective parameterisations of the reflection subgroups of affine Weyl groups on the nose, i.e., up to equality as subsets. Both parameterisations are in terms of explicitly described combinatorial objects attached to finite root systems, and their proofs do not use the classification of finite or affine Weyl groups. Their parameterisation in 4 , by specifying their "canonical simple system", generalises the well-known classification of reflection subgroups of the infinite dihedral group by specifying a set of generating reflections of minimal total length. The parameterisation in \$5. by explicitly describing their root system, generalises the classification of nontrivial reflection subgroups of the infinite dihedral group by specifying, for each such subgroup, its set of reflections in terms of a coset in $\mathbb{Z}$ of a subgroup of $\mathbb{Z}$. We indicate how to describe the root system and conjugacy action, and to compute the index, etc. in terms of these parameters. Some of these applications overlap somewhat with results in [13], [14], [12, for example, but our case-free approach to them is quite different. One or the other of our parametrisations is 
typically more suitable for any particular application; the relation between the two parameterisations is considered in detail in 6 .

We make a final remark as to applications of our results. A Lie subalgebra $\mathfrak{g}_{1}$ of a complex semisimple or Kac-Moody Lie algebra $\mathfrak{g}$ is said to be regular if it is invariant under a Cartan subalgebra $\mathfrak{h}$ of $\mathfrak{g}$. The root system of a regular subalgebra $\mathfrak{g}_{1}$ with respect to $\mathfrak{h}$ may naturally be regarded as a closed subsystem of that of $\mathfrak{g}$. The regular semisimple subalgebras of a simple complex Lie algebra were classified in [11, Ch. 2, §5] for example (hence the emphasis on closed subsystems there). Similarly, [13, §5] determines the possible types of regular Kac-Moody subalgebras of a (twisted or untwisted) affine Kac-Moody Lie algebra $\mathfrak{g}$. Though we do not go into this here, our classifications of reflection subgroups up to equality may be applied to the classification up to equality, and the study of the structure of $\mathfrak{h}$ invariant regular Kac-Moody subalgebras of $\mathfrak{g}$ of the type investigated in [13, for fixed $\mathfrak{h}$.

\section{The CASE OF A Finite Root SYSTEM}

We begin with the following well-known characterisation of the simple subsystems of $\Phi$.

Lemma 1. Let $\Phi$ be a finite root system in the Euclidean space $V$. A subset $\Gamma \subseteq \Phi$ is a simple subsystem of $\Phi$ (i.e. a simple system in a root subsystem of $\Phi$ ) if and only if

(i) $\Gamma$ is linearly independent.

(ii) For any pair $\alpha, \beta \in \Gamma,\left\langle\alpha, \beta^{\vee}\right\rangle \leq 0$.

Proof. If $\Gamma$ is a simple subsystem of $\Psi \subseteq \Phi$, then by definition $\Gamma$ is linearly independent, and the property (ii) is standard (cf. [27, Appendix, Lemma (10)]).

Conversely, let $\Gamma \subseteq \Phi$ satisfy conditions (i) and (ii) and write $W^{\prime}$ for the group generated by the reflections $s_{\gamma}(\gamma \in \Gamma)$. Let $\Psi$ be the set of roots $W^{\prime} \Gamma \subseteq \Phi$. Then $\Psi$ is a crystallographic root system with Weyl group $W^{\prime}$, for if $\alpha \in \Psi, \alpha=w \gamma$ for some $w \in W^{\prime}$ and $\gamma \in \Gamma$. Hence $s_{\alpha}=s_{w \gamma}=w s_{\gamma} w^{-1} \in W^{\prime}$, and so $\Psi$ is precisely the set of roots $\alpha \in \Phi$ such that $s_{\alpha} \in W^{\prime}$. Replacing $W^{\prime}$ with $W$ and $\Psi$ with $\Phi$, we may therefore suppose that the reflections $s_{\gamma}(\gamma \in \Gamma)$ generate $W$ and that $\Phi=W \Gamma$.

Let $\Phi_{+}$be the roots of $\Phi$ which are positive linear combinations of $\Gamma$. If $\dot{\alpha}$ denotes the unit vector in the direction of $\alpha$, the assumptions imply that if $\gamma_{1}, \gamma_{2} \in \Gamma$, then $\left\langle\dot{\gamma}_{1}, \dot{\gamma}_{2}\right\rangle \in\left\{-\cos \frac{\pi}{2},-\cos \frac{\pi}{3},-\cos \frac{\pi}{4},-\cos \frac{\pi}{6}\right\}$. It now follows from [8, Lemma (4.2)] that $\Phi=\Phi_{+} \amalg-\Phi_{+}$.

We shall also require the following result.

Lemma 2. Let $\Phi$ be an indecomposable finite crystallographic root system in the Euclidean space $V$, and let $\Gamma \subseteq \Phi$ be a simple subsystem of $\Phi$. Let $\mathcal{C}$ be the corresponding (closed) fundamental chamber for the action of $W=W(\Phi)$ on $V$. Then $\alpha \in \mathcal{C} \cap \Phi$ if and only if $\alpha$ is the highest root $\omega$ of $\Phi$ or $\alpha=\omega^{* \vee}$, where $\omega^{*}$ is the highest root of $\Phi^{\vee}$.

Proof. It follows from arguments based on the fact that for any two roots $\alpha_{1}, \alpha_{2} \in$ $\Phi$, we have $\left\langle\alpha_{1}, \alpha_{2}\right\rangle\left\langle\alpha_{2}, \alpha_{1}\right\rangle=4 \cos ^{2} \phi$, where $\phi$ is the angle between $\alpha_{1}$ and $\alpha_{2}$, that there are at most two possibilities for the length of a root in $\Phi$ (see [2, Ch. VI, §1.3]). 
It is also standard that $W$ acts transitively on the set of roots of a given length. Now it is clear that the roots $\omega, \omega^{* \vee}$ of the statement lie in $\mathcal{C}$, and by the regularity of the action of $W$ on the set of chambers, they are the only roots in their $W$ orbit in $\mathcal{C}$. But the highest root is always long, whence the $W$-orbit of $\omega$ and $\omega^{* \vee}$ include all roots of $\Phi$. This proves the lemma.

Note that in the above lemma, $\omega$ and $\omega^{* \vee}$ have the same length (and hence coincide) if and only if $\Phi$ has just one root length. In that case, we declare all roots of $\Phi$ to be both short and long.

The root subsystem $\Psi \subseteq \Phi$ is said to be parabolic if $\Phi$ has a simple system $\Pi$ such that there is a subset $\Gamma \subseteq \Pi$ which is a simple system for $\Psi$. The parabolic subgroups (i.e. the Weyl groups of parabolic subsystems) are known to be precisely the stabilisers in $W$ of points of $V$. In general $W$ will have reflection subgroups other than parabolic ones; the next definition provides a convenient way of describing them.

Definition 2. Let $\Psi \subsetneq \Phi$ be a root subsystem. We say that $\Phi$ is an elementary extension of $\Psi$ if there is a simple system $\Pi$ and an indecomposable component $\Sigma$ of $\Phi$ such that a simple system for $\Psi$ is obtained by deleting one element of $\Sigma$ from $\Pi \cup\{-\theta\}$, where $\theta$ is a root of $\Sigma$ which lies in the chamber $\mathcal{C}$ defined by the positive system corresponding to $\Pi$.

By Lemma 2, $\theta$ is the highest root of $\Sigma$ or $\theta^{\vee}$ is the highest root of $\Sigma^{\vee}$. We shall prove

Theorem 1 (cf. $\$ 1.3$ and Appendix $\AA$ below). Let $\Phi$ be a finite crystallographic root system in the Euclidean space $V$, and let $\Psi \subsetneq \Phi$ be a root subsystem. Then there is a root subsystem $\widehat{\Psi}$ with $\Psi \subsetneq \widehat{\Psi} \subseteq \Phi$, such that either $\Psi$ is parabolic in $\widehat{\Psi}$ or $\widehat{\Psi}$ is an elementary extension of $\hat{\Psi}$.

Proof. Let $\Gamma$ be a simple system for $\Psi$, with corresponding positive system $\Psi_{+} \subseteq \Psi$. Denote the Weyl group of $\Psi$ by $W^{\prime}$, and let $\mathcal{C}=\{v \in V \mid\langle\gamma, v\rangle \geq 0$ for $\gamma \in \Gamma\}$ be the fundamental chamber for the action of $W^{\prime}$ corresponding to the simple system $\Gamma$.

Since $\Psi \neq \Phi$, there is a root $\alpha \in \Phi \backslash \Psi$. There is a $W^{\prime}$-transform $w \alpha \in-\mathcal{C}$ $\left(w \in W^{\prime}\right)$, and since clearly $w \alpha \notin \Psi$, we may assume that $\alpha \in-\mathcal{C}$. It follows that $\langle\alpha, \gamma\rangle \leq 0$ for all $\gamma \in \Gamma$.

Let $\underset{\widetilde{\Gamma}}{\bar{T}}=\Gamma \cup\{\alpha\}$. If $\widetilde{\Gamma}$ is linearly independent, then by Lemma 1, $\widetilde{\Gamma}$ is a simple subsystem of $\Phi$, and taking $\widehat{\Psi}$ to be the root system corresponding to $\widetilde{\Gamma}$, clearly $\Psi$ is a parabolic subsystem of $\widehat{\Psi}$.

Suppose then that $\widetilde{\Gamma}$ is linearly dependent. As with any set of roots, $\widetilde{\Gamma}$ has a unique partition

$$
\widetilde{\Gamma}=\Gamma_{1} \cup \Gamma_{2} \cup \cdots \cup \Gamma_{k},
$$

where the $\Gamma_{i}$ are mutually orthogonal (i.e. if $\gamma_{i} \in \Gamma_{i}$ and $i \neq j$, then $\left\langle\gamma_{i}, \gamma_{j}\right\rangle=0$ ) and cannot be further decomposed as in (2).

Evidently $\alpha$ lies in precisely one of the sets $\Gamma_{i}$, and we may suppose $\alpha \in \Gamma_{1}$. Now the matrix of pairwise inner products of the elements of $\Gamma_{1}$ satisfy the conditions of [2, Ch. V, §3, Lemma 5]. Hence there are positive real numbers $c_{\gamma}$ such that $\sum_{\gamma \in \Gamma_{1}} c_{\gamma} \gamma=0$, and the $c_{\gamma}$ are uniquely determined, up to a common multiple, 
and for all $\gamma, c_{\gamma} \neq 0$. It follows from Lemma 1 that every proper subset of $\Gamma_{1}$ is a simple subsystem of $\Phi$.

Now the matrix $\left(\left\langle\gamma, \delta^{\vee}\right\rangle\right)_{\gamma, \delta \in \Gamma_{1}}$ is an indecomposable generalised Cartan matrix of affine type in the sense of [4, \$15.3]. These have been completely classified (see, e.g. op. cit. $\S \S 15.3,17.1$ ), and the following statement is an easy consequence of this classification (for an alternative proof using affine Weyl groups, and independent of the classification, see Corollary $3(b)$ ).

There is an element $\theta \in \Gamma_{1}$ such that if $\Gamma_{1}^{\prime}=\Gamma_{1} \backslash\{\theta\}$, then

(i) $\Gamma_{1}^{\prime}$ is a simple system for an indecomposable root system $\Psi_{1}^{\prime}$, and

(ii) $\theta \in \Psi_{1}^{\prime}$.

By Lemma 2, $\theta$ is the negative of either the highest root of $\Psi_{1}$ or of the coroot of the highest root of $\Psi_{1}^{\vee}$.

Let $\widehat{\Psi}=\Psi_{1}^{\prime} \cup \Psi_{2} \cup \cdots \cup \Psi_{k}$, where $\Psi_{1}^{\prime}$ is as above, and for $i=2, \ldots, d, \Psi_{i}$ is the root subsystem with simple system $\Gamma_{i}$. Then $\widehat{\Psi}$ is evidently an elementary extension of $\Psi$ with the desired properties.

The theorem above leads to the following algorithm for describing all isomorphism types of reflection subgroups of a Weyl group. It depends on the Dynkin diagram and its completion, whose definition (see [2, Ch. 6, §4, no. 2-3] and [21, $\S 4.7])$ we now recall.

For any two roots $\alpha, \beta$ in a root system $\Phi$, we have $n_{\alpha, \beta}:=\left\langle\alpha, \beta^{\vee}\right\rangle=\frac{2\langle\alpha, \beta\rangle}{\langle\beta, \beta\rangle} \in \mathbb{Z}$. Let $\Gamma$ be a subset of $\Phi$ such that $n_{\alpha, \beta} \leq 0$ for all distinct pairs $\alpha, \beta \in \Gamma$. The diagram $\Delta(\Gamma)$ associated to $\Gamma$ is a graph with nodes the elements of $\Gamma$ and an edge of valency $\max \left(-n_{\alpha, \beta},-n_{\beta, \alpha}\right)$ between any two distinct $\alpha, \beta \in \Gamma$. Further, if the valency $-n_{\alpha, \beta}>1$, one attaches to the edge between $\alpha$ and $\beta$ an arrowhead $(>)$ pointing towards $\beta$. For example, if $\alpha=-\beta$, there is a double edge equipped with two arrowheads pointing in opposite directions joining $\alpha$ and $\beta$.

Now consider any root system $\Phi$. Choose a simple system $\Gamma$ of $\Phi$. The Dynkin diagram of $\Phi$ is $\Delta(\Gamma)$. If $\Phi$ is indecomposable, the completed Dynkin diagram of $\Phi$ is defined as $\Delta\left(\Gamma \amalg\left\{-\alpha_{h}\right\}\right)$, where $\alpha_{h}$ is the highest root of $\Phi$ with respect to $\Gamma$. Regarded as edge-marked graphs, i.e. as graphs whose edges of valency $>1$ are marked with arrowheads, they are independent of the choice of a simple system. Note that the nodes of these diagrams are not labelled by specific roots.

Corollary 1. Let $\Phi$ be a finite crystallographic root system in the Euclidean space $V$. The Dynkin diagram of any root subsystem of $\Phi$ is obtained by a sequence of operations of the following type on Dynkin diagrams, beginning with the Dynkin diagram of $\Phi$ :

(1) Delete any number (possibly zero) of nodes.

(2) Replace some indecomposable component $\Sigma$ of the Dynkin diagram by the completed Dynkin diagram of $\Sigma$ or $\Sigma^{\vee}$, and delete at least one node from the resulting affine component.

Proof. If $\Psi$ is a parabolic subsystem of $\Phi$, its Dynkin diagram is obtained from that of $\Phi$ by deleting nodes. By Theorem 1, we need only relate the Dynkin diagram of a subsystem $\Psi$ of $\Phi$ to that of $\Phi$ when $\Phi$ is an elementary extension of $\Psi$. In that case, taking into account Lemma 2, the result follows from the nature of an elementary extension, given that a root system and its dual have the same (unoriented) Dynkin diagram. 
It is well known that any parabolic subsystem of $\Phi$ is closed in $\Phi$. Also, it follows from the definitions and the results of Borel and de Siebenthal 21] that if $\Phi$ is an elementary extension of $\Psi$, then either $\Psi$ is closed in $\Phi$ or $\Psi^{\vee}$ is closed in $\Phi^{\vee}$. Hence we have the following:

Corollary 2. If $\Psi$ is a maximal proper root subsystem of $\Phi$, then either $\Psi$ is closed in $\Phi$ or $\Psi^{\vee}$ is closed in $\Phi^{\vee}$.

Given Corollary [2, Theorem 11 and Corollary 1] are easily deduced from the results of Dynkin [11] or Borel and de Siebenthal [21]. However, we do not know any independent simple proof of Corollary 2

\section{Affine Weyl groups}

3.1. Background. We introduce some notation and present a summary of the necessary background on affine Weyl groups, most of which may be found in 2, Ch. VI, §2] or [20, Ch. 6-7].

Let $Z$ be any additive subgroup of $\mathbb{R}$ (typically $Z=\mathbb{Z}$ or $Z=\mathbb{R}$ in applications). Let $\Psi$ be any root system in $V$. We define $Q_{Z}(\Psi)$ to be the additive subgroup of $V$ generated by elements $z \alpha^{\vee}$ for $z \in Z$ and $\alpha \in \Psi$. We define $P_{Z}(\Psi)$ as the additive subgroup

$$
P_{Z}(\Psi):=\left\{v \in \mathbb{R} \Psi \mid\left\langle v, \alpha^{\vee}\right\rangle \in Z \text { for all } \alpha \in \Psi\right\}
$$

of $V$. Let $\Gamma$ be any simple system of $\Psi$. Define the corresponding fundamental coweights $\omega_{\gamma}=\omega_{\gamma}(\Gamma) \in \mathbb{R} \Psi$ of $\Psi$, for $\gamma \in \Gamma$, by $\left\langle\gamma, \omega_{\gamma^{\prime}}\right\rangle=\delta_{\gamma, \gamma^{\prime}}$. Then $Q_{Z}(\Psi)=$ $\bigoplus_{\alpha \in \Gamma} Z \alpha^{\vee} \cong Z^{|\Gamma|}$ and $P_{Z}(\Psi)=\bigoplus_{\alpha \in \Gamma} Z \omega_{\alpha} \cong Z^{|\Gamma|}$.

The cases $P(\Psi):=P_{\mathbb{Z}}(\Psi)$ and $Q(\Psi):=Q_{\mathbb{Z}}(\Psi)$ are of special significance. These are the the coweight lattice and the coroot lattice of $\Psi$ (under the natural identifications of the dual space of $\mathbb{R} \Psi$ with $\mathbb{R} \Psi$ induced by $\langle-,-\rangle)$. We shall sometimes write $P(\Gamma)$ for $P(\Psi)$ and $Q(\Gamma)$ for $Q(\Psi)$.

The index $[P(\Gamma): Q(\Gamma)]$ is called the index of connection ([2, Ch. VI, $\S 1$, no. 9]) of $\Psi$ (or $\Gamma)$ and will be denoted as $f_{\Psi}$ or $f_{\Gamma}$. It is known ([2, Ch. VI, §1, Ex. 7]) that

$$
f_{\Gamma}=\operatorname{det}\left(\left(\left\langle\alpha, \beta^{\vee}\right\rangle\right)_{\alpha, \beta \in \Gamma}\right)
$$

is equal to the determinant of the Cartan matrix of $\Psi$.

3.2. For the remainder of 33 , we take $\Phi$ to be a reduced finite crystallographic root system in $V$, as in $\$ 1$ For simplicity, assume $\Phi$ spans $V$. Write $P=P(\Phi)$ and $Q=Q(\Phi)$. For $v \in V$, denote by $\tau_{v}: V \rightarrow V$ the translation $x \mapsto x+v$. For any subgroup $B$ of $V$, let $\tau_{B}:=\left\{\tau_{\alpha} \mid \alpha \in B\right\}$ denote the group of translations of $V$ by elements of $B$.

For $\alpha \in \Phi$ and $m \in \mathbb{Z}$, define the affine reflection

$$
s_{\alpha, m}(v)=v-(\langle\alpha, v\rangle-m) \alpha^{\vee}=s_{\alpha}(v)+m \alpha^{\vee} .
$$

We let $H_{\alpha, m}:=\left\{v \in V \mid s_{\alpha, m}(v)=v\right\}=\{v \in V \mid\langle v, \alpha\rangle=m\}$ denote the reflecting hyperplane of $s_{\alpha, m}$.

Definition 3. The affine Weyl group $W^{a}$ associated to $\Phi$ is the group generated by $\left\{s_{\alpha, m} \mid \alpha \in \Phi, \quad m \in \mathbb{Z}\right\}$. The extended affine Weyl group $\widetilde{W}^{a}$ is the group generated by $W \cup \tau_{P}$. The alcoves of $W^{a}$ are the connected components (for the standard topology of $V$ ) of $V \backslash \bigcup_{\alpha \in \Phi, n \in \mathbb{Z}} H_{\alpha, n}$. A special point of $W^{a}$ is a point of $V$ which for each $\alpha \in \Phi$ lies in some reflecting hyperplane $H_{\alpha, m}(m \in \mathbb{Z})$. 
Proposition 1. (a) The group $W^{a}$ is the semidirect product $W \ltimes \tau_{Q}$ of $W$ and $\tau_{Q}$, while $\widetilde{W}^{a}$ is the semidirect product $W \ltimes \tau_{P}$ of $W$ and $\tau_{P}$. Moreover, $W^{a}$ is a normal subgroup of $\widetilde{W}^{a}$ (i.e. $W^{a} \unlhd \widetilde{W}^{a}$ ) such that $\widetilde{W}^{a} / W^{a} \simeq P / Q$.

(b) Let $\Pi$ be a simple system of $\Phi$. Then $W^{a}$ is a Coxeter group, with Coxeter generators $S^{a}:=\left\{s_{\alpha} \mid \alpha \in \Pi\right\} \cup\left\{s_{\theta, 1} \mid \theta \in H\right\}$, where $H$ is the set of highest roots of the indecomposable components of $\Phi$ (with respect to their simple systems contained in $\Pi)$.

(c) Let $A:=\{v \in V \mid\langle v, \alpha\rangle \geq 0$ for all $\alpha \in \Pi, \quad\langle v, \theta\rangle \leq 1$ for all $\theta \in H\}$. Then $A$ is the closure of the fundamental alcove $A^{0}$ for $\left(W^{a}, S^{a}\right)$ on $V$. In particular, $A$ is the closure of $A^{0}, A^{0}$ is the interior of $A$, and $S^{a}$ is the set of reflections in the walls $\left\{H_{\alpha, 0} \mid \alpha \in \Pi\right\} \cup\left\{H_{\theta, 1} \mid \theta \in H\right\}$ of $A$.

(d) Each closed alcove contains at least one special point of $W^{a}$. The set of all special points coincides with the coweight lattice of $\Phi$.

(e) The indecomposable components of $W^{a}$ are the affine Weyl groups associated to the indecomposable components of $\Phi$, and the group $\widetilde{W}^{a}$ is the direct product of the extended affine Weyl groups of the indecomposable components of $\Phi$.

Remark 1. See [2, Ch. V, §1-3] for more details on alcoves, walls, special points, etc. For indecomposable $\Phi$, the above facts can be found in [2]. For decomposable $\Phi$, the statements are either in [2] or follow readily from the results there about decomposable affine Weyl groups. It is well known that any closed alcove is a fundamental domain for the action of $W^{a}$ on $V$. In this paper, by a fundamental domain for a group acting on a set, we shall simply mean a set of orbit representatives, though our fundamental domains will usually satisfy additional properties such as connectedness.

3.3. Linear realisation. The groups $W^{a}$ and $\widetilde{W}^{a}$ appear above as groups of affine transformations of $V$. They may also be viewed as groups of linear transformations of a space $\widehat{V}$ which contains $V$ as a subspace of codimension 1 . In particular, we shall see that there is an affine root system in $\widehat{V}$ on which both $\widetilde{W}^{a}$ and $W^{a}$ act naturally.

Let $\widehat{V}=V \oplus \mathbb{R} \delta$, where $\delta$ is an indeterminate. The inner product $\langle-,-\rangle$ on $V$ has a unique extension to a symmetric bilinear form on $\widehat{V}$ which is positive semidefinite and has a radical equal to the subspace $\mathbb{R} \delta$. This extension to $\widehat{V}$ will also be denoted by $\langle-,-\rangle$. For any non-isotropic element $v \in \widehat{V}$, let $\widehat{s}_{v}: \widehat{V} \rightarrow \widehat{V}$ denote the $\mathbb{R}$-linear map $x \mapsto x-\left\langle x, v^{\vee}\right\rangle v$ where $v^{\vee}=\frac{2}{\langle v, v\rangle} v$. For any $v \in V$, let $t_{v}: \widehat{V} \rightarrow \widehat{V}$ denote the $\mathbb{R}$-linear map $x \mapsto x+\langle x, v\rangle \delta$.

Let $\Pi$ be a simple system of $\Phi(\subset V \subset \widehat{V})$ with a corresponding set $\Phi_{+}$of positive roots.

Definition 4. Define the following subsets of $\widehat{V}$ :

$$
\begin{aligned}
& \Phi^{a}:=\Phi+\mathbb{Z} \delta, \\
& \widehat{\Pi}:=\Pi \cup\{-\theta+\delta \mid \theta \in H\}, \text { and } \\
& \Phi_{+}^{a}:=\left\{\alpha+n \delta \mid \alpha \in \Phi, n \in \mathbb{Z}_{\geq 0}, n>0 \text { if } \alpha \in-\Phi_{+}\right\} .
\end{aligned}
$$

Proposition 2. (a) The group $\widetilde{W}^{a}$ (resp., $W^{a}$ ) is isomorphic to the group generated by $W$ and $t_{\gamma}$ for $\gamma \in P$ (resp., $\gamma \in Q$ ). Under this isomorphism, 
$t_{\gamma}$ is mapped to $\tau_{-\gamma}$, and for $\alpha \in \Phi$ and $m \in \mathbb{Z}, s_{\alpha, m}$ is mapped to $\widehat{s}_{\alpha-m \delta}=$ $\widehat{s}_{m \delta-\alpha}$.

(b) We have that $\Phi^{a}$ is $\widetilde{W}^{a}$-stable, $W^{a}=\left\langle\widehat{s}_{\alpha} \mid \alpha \in \Phi^{a}\right\rangle, \Phi^{a}=W^{a} \widehat{\Pi}=$ $\Phi_{+} \dot{\cup}-\Phi_{+}$, and $\Phi_{+}=\Phi \cap \mathbb{R}_{\geq 0} \widehat{\Pi}$. Moreover, the set $S^{a}$ of Proposition 1 is mapped to $\left\{\widehat{s}_{\alpha} \mid \alpha \in \widehat{\Pi}\right\}$.

Proof. Let $W_{1}^{a}$ (resp., $\widetilde{W}_{1}^{a}$ ) denote the group of linear endomorphisms of $\widehat{V}$ defined in (a).

The proof will use the following elementary formulae concerning the action of these groups on $\widehat{V}$, which are listed here for future reference:

$$
\begin{gathered}
\widehat{s}_{\alpha} t_{\gamma}=t_{\widehat{s}_{\alpha}(\gamma)} \widehat{s}_{\alpha}, \quad \text { for } \alpha \in \Phi^{a}, \gamma \in \widehat{V}, \\
\widehat{s}_{\alpha+n \delta}=t_{n \alpha^{\vee}} \widehat{s}_{\alpha}, \quad \text { for } \alpha \in \Phi, n \in \mathbb{Z}, \\
\widehat{s}_{\alpha+n \delta} \widehat{s}_{\alpha+m \delta}=t_{(n-m) \alpha^{\vee}}, \quad \text { for } \alpha \in \Phi, n, m \in \mathbb{Z}, \\
\widehat{s}_{\alpha+n \delta}(\beta+m \delta)=s_{\alpha}(\beta)+\left(m-n\left\langle\beta, \alpha^{\vee}\right\rangle\right) \delta \quad \text { for } \alpha, \beta \in \Phi \text { and } n, m \in \mathbb{Z}, \\
w t_{\gamma}(\alpha+m \delta)=w(\alpha)+(m+\langle\alpha, \gamma\rangle) \delta \quad \text { for } w \in W, \gamma \in V, \alpha \in \Phi, m \in \mathbb{Z} .
\end{gathered}
$$

Consider the contragredient action of $\widetilde{W}_{1}^{a}$ and $W_{1}^{a}$ on the dual space

$$
\widehat{V}^{*}:=\operatorname{Hom}_{\mathbb{R}}(\widehat{V}, \mathbb{R}),
$$

defined by $w f=f \circ w^{-1}$ for $f \in \widehat{V}^{*}$ and $w \in \widehat{W}^{a}$. Define $d \in \widehat{V}^{*}$ by $d(v+c \delta)=c$ for $v \in V, c \in \mathbb{R}$. The affine map $i: V \rightarrow \widehat{V}^{*}$, defined by $i(v)(x)=\langle v, x\rangle+d(x)$ for $v \in V$ and $x \in \widehat{V}$, is injective and has an image equal to the affine hyperplane $E=\left\{f \in \widehat{V}^{*} \mid f(\delta)=1\right\}$ of $\widehat{V}^{*}$. This is $\widetilde{W}_{1}^{a}$-stable since $\widetilde{W}_{1}^{a}$ fixes $\delta$. Since $E$ spans $\widehat{V}^{*}$ as a real vector space, restriction to $E$ defines an isomorphism of $\widetilde{W}_{1}^{a}$ (and hence of $W_{1}^{a}$ ) with a group of affine transformations of $E$. But from the definitions, one readily checks that

$$
t_{\gamma} i=i \tau_{-\gamma} \quad \text { for } \gamma \in V
$$

and

$$
\widehat{s}_{\alpha} i=i s_{\alpha} \quad \text { for } \alpha \in \Phi \text {. }
$$

The first assertion of (a) follows by noting that by (11) and (12), $i: V \rightarrow E$ is an isomorphism of $\widetilde{W}^{a}$-modules. We also observe that under the above map, the element $s_{\alpha, m}=\tau_{m \alpha^{\vee}} s_{\alpha}$ of $W^{a}$ is mapped to $t_{-m \alpha^{\vee}} \widehat{s}_{\alpha}=\widehat{s}_{m \delta-\alpha}$ in $\widehat{W}_{1}^{a}$. This completes the proof of (a).

It is not difficult to prove (b) using the above formulae and well-known facts about root systems of Weyl groups. Instead, we simply observe that the proof of (b) reduces easily to the special case in which $\Phi$ is indecomposable, in which case $\Phi^{a}$ may be identified with the system of real roots of an untwisted affine Kac-Moody Lie algebra [20, Prop. 6.3(a)], and given that $\Phi^{a}$ is $\widehat{W}^{a}$-stable, the claims of (b) are well known (see [20, Ch. 6-7]). To see that $\Phi^{a}$ is $\widehat{W}^{a}$-stable, it is clear that $\Phi^{a}$ is stable under $W^{a}$, and to prove stability under $\widehat{W}^{a}$, we apply (10) with $\gamma$ in the coweight lattice and note that $\langle\alpha, \gamma\rangle \in \mathbb{Z}$. 
3.4. Let $R$ be a fixed subgroup of $P$ containing $Q$. It is well known that $R$ is $W$-stable, so one may form the semidirect product $\widetilde{W}:=W \ltimes \tau_{R}$ which satisfies $W^{a} \subseteq \widetilde{W} \subseteq \widetilde{W^{a}}$ and $\left[\widetilde{W}: W^{a}\right]=[R: Q]$. Many of our results for $\widetilde{W}^{a}$ apply equally to any of the subgroups $\widetilde{W}$.

For $v \in V \subset \widehat{V}$, we have $\widehat{s}_{v}=s_{v, 0} \oplus \operatorname{id} d_{\mathbb{R} \delta}=s_{v} \oplus \operatorname{id} \mathbb{R}_{\mathbb{R} \delta}$. Henceforth, we shall therefore write $s_{\alpha}$ instead of $\widehat{s}_{\alpha}$ for any $\alpha \in \Phi$. We shall regard $\widetilde{W}^{a}, W^{a}$ and $\widetilde{W}$ as groups of linear transformations of $\widehat{V}$ or as groups of affine transformations of $V$, as convenience dictates.

Let $\Delta \subseteq \widehat{V} \backslash \mathbb{R} \delta$. The (indecomposable) components of $\Delta$ are the inclusionminimal non-empty subsets $\Delta^{\prime}$ of $\Delta$ which are orthogonal to their complement in $\Delta$. Say that $\Delta$ is indecomposable if it has only one component. If $\Delta$ is a simple subsystem or a root subsystem of $\Phi^{a}$, these coincide with the usual notions of indecomposable components and indecomposability.

3.5. Linear versus affine. This subsection and the next two describe relationships between $\Phi^{a}$ and root systems of affine Weyl groups, and the realisation of $W^{a}$ as a group of affine transformations in a smaller space. It will turn out that comparison of the two realisations of affine Weyl groups as linear groups and as affine linear groups can be effectively exploited.

Recall ([2, Ch. IV, §1.9]) that for any set $I$, an $I$-indexed Coxeter matrix is any symmetric matrix of the form $M=\left(m_{i, j}\right)_{i, i \in I}$, where $m_{i j} \in \mathbb{Z}_{>0} \amalg\{\infty\}$, and $m_{i j}=1$ if and only if $i=j$. Let $M$ be such a Coxeter matrix. The Coxeter system $\left(W_{M}, S_{M}\right)$ with Coxeter matrix $M$ has a standard faithful representation, the "Tits representation", as a reflection group of isometries of a space $U$ with basis $e_{i}$ for $i \in I$. This representation is described in [2, Ch. V, §4] and [18, 5.3-5.4]. Denote by $B_{M}$ the matrix $B_{M}=\left(-\cos \frac{\pi}{m_{i, j}}\right)_{i, j \in I}$. The space $U$ has a symmetric $\mathbb{R}$-valued bilinear form $(-,-)$ satisfying $\left(\left(e_{i}, e_{j}\right)\right)_{i, j \in I}=B_{M}$. The simple reflections $S_{M}$ are the orthogonal reflections $r_{i}$ on $U$ whose fixed hyperplanes are the $e_{i}^{\perp}(i \in I)$; $W_{M}$ is then the group of linear transformations of $U$ generated by $S_{M}$. There is an associated root system $\Phi_{M}$ for $\left(W_{M}, S_{M}\right)$ defined by $\Phi_{M}=W_{M} \Pi_{M}$, where $\Pi_{M}=\left\{e_{i} \mid i \in I\right\}$.

3.6. Now take $\widehat{V}=V \oplus \mathbb{R} \delta$ as above, and note that with respect to the form $\langle-,-\rangle$, the set of isotropic elements of $\widehat{V}$ is $\mathbb{R} \delta$. For any non-isotropic $\alpha \in \widehat{V}$, we set $\dot{\alpha}:=\frac{1}{\|\alpha\|} \alpha$, where $\|\alpha\|:=\langle\alpha, \alpha\rangle^{1 / 2}$. Define the map $\iota: \widehat{V} \backslash \mathbb{R} \delta \rightarrow \widehat{V} \backslash \mathbb{R} \delta$ by $\iota(\alpha)=\dot{\alpha}$. For any subset $\Gamma$ of $\widehat{V} \backslash \mathbb{R} \delta$, we set $\Gamma^{\vee}:=\left\{\alpha^{\vee} \mid \alpha \in \Gamma\right\}$ and $\dot{\Gamma}:=\iota(\Gamma)=\{\dot{\alpha} \mid \alpha \in \Gamma\}$.

Remark 2. If $M$ is the Coxeter matrix of $\left(W^{a}, S^{a}\right)$ and $W^{a}$ is indecomposable, then there is an identification $U=\widehat{V}$ (as real vector spaces with symmetric bilinear forms) such that $\Phi_{M}=\dot{\hat{\Psi}}, \Pi_{M}=\dot{\hat{\Pi}}$ and $\left(W^{a}, S^{a}\right)=\left(W_{M}, S_{M}\right)$. This is not true if $W^{a}$ is decomposable, since $\widehat{\Pi}$ is then linearly dependent, and $\widehat{V}$ is obtained from $U$ by taking the quotient by an appropriate subspace of the radical of $U$. The advantage of the root system $\Phi^{a}$ is that its root subsystems may be treated in a similar way to $\Phi^{a}$ itself, since even for indecomposable $\Phi$, root subsystems of $\Phi^{a}$ need not have linearly independent simple roots. 
3.7. It is evident from the proof of Proposition 2 that the indecomposable components of the root system $\Phi^{a}$ may be identified with the real root systems of untwisted affine Kac-Moody Lie algebras associated to the components of $\Phi$ [20]. If $\Phi$ is not indecomposable, then $\widehat{\Pi}$ is not linearly independent. However, $\widehat{\Pi}$ is positively independent in the following sense: a subset $\Sigma$ of an $\mathbb{R}$-vector space is said to be positively independent if $\sum_{\gamma \in \Sigma} c_{\gamma} \gamma=0$ with all $c_{\gamma} \geq 0$, and almost all $c_{\gamma}=0$ implies that all $c_{\gamma}=0$. It follows that $(\widehat{V},\langle-,-\rangle, \dot{\hat{\Pi}})$ is a based root datum with root system $\dot{\Phi^{a}}$ in the sense of $[9]$ with positive roots $\dot{\Phi^{a}}{ }_{+}=\iota\left(\Phi_{+}^{a}\right)$ and associated Coxeter system $\left(W^{a}, S^{a}\right)$. This is enough to ensure that standard facts about root systems of Coxeter groups apply to $\Phi^{a}$; see 9 for properties of based root systems and 6], 24] for discussions of root systems closely related to those considered here.

3.8. The fact proved below will play an important role in this paper. Let $M=$ $\left(m_{\alpha \beta}\right)_{\alpha, \beta \in \Gamma}$ be a Coxeter matrix where $\Gamma$ is a finite set, and let

$$
B_{M}:=\left(-\cos \frac{\pi}{m_{\alpha \beta}}\right)_{\alpha, \beta \in \Gamma}
$$

be the matrix of the invariant form on the space of the Tits representation of the corresponding Coxeter group.

Proposition 3. Suppose that $\Gamma \subseteq V \backslash\{0\}$ is indecomposable and linearly dependent, and that the matrix $B:=(\langle\dot{\alpha}, \dot{\beta}\rangle)_{\alpha, \beta \in \Gamma}$ is equal to $B_{M}$ for some $\Gamma$-indexed Coxeter matrix $M$ (cf. 3.5). Then there exists $\theta \in \Gamma$ and scalars $c_{\gamma} \in \mathbb{R}_{>0}$ for $\gamma \in \Gamma$ with the following property: the set $\Lambda:=\left\{c_{\gamma} \gamma \mid \gamma \in \Gamma \backslash\{\theta\}\right\}$ is the set of simple roots of an indecomposable, reduced crystallographic root system $\Psi$, and $-c_{\theta} \theta$ is the highest root of $\Psi$ with respect to $\Lambda$. Moreover, the affine Weyl group of $\Psi$ is naturally isomorphic to $W_{M}$, and the set $\left\{c_{\gamma}\right\}_{\gamma \in \Gamma}$ is uniquely determined up to multiplication by a positive scalar.

Proof. Consider the Coxeter matrix $M=\left(m_{\alpha, \beta}\right)_{\alpha, \beta \in \Gamma}$ and the associated reflection representation of $\left(W_{M}, S_{M}\right)$ in $U$, an $\mathbb{R}$-vector space with basis $e_{\alpha}$ for $\alpha \in \Gamma$, as described in 3.5. Set $\Delta:=\left\{e_{\alpha} \mid \alpha \in \Gamma\right\}$. We have by construction that

$$
\left(\left(e_{\alpha}, e_{\beta}\right)\right)_{\alpha, \beta \in \Gamma}=B \text {. }
$$

If $\alpha \neq \beta$, then $\left(e_{\alpha}, e_{\beta}\right)=-\cos \frac{\pi}{m_{\alpha \beta}} \leq 0$. It follows from [2, Ch. V, $\S 3$, Lemme 5] that the subspace $\mathbb{R} \Gamma$ has dimension $|\Gamma|-1$ and there is a linear relation $\sum_{\alpha \in \Gamma} c_{\alpha} \alpha=$ 0 which is unique up to multiplication by a non-zero scalar. Moreover, this relation may be uniquely chosen so that $c_{\alpha}>0$ and $\sum_{\alpha \in \Gamma} c_{\alpha}=1$. Hence in particular our assumptions imply that the matrix $B$ is positive semidefinite of corank 1 .

The linear map $L: U \rightarrow V$ defined by $e_{\alpha} \mapsto \dot{\alpha}$ for $\alpha \in \Gamma$ satisfies $\left\langle L(u), L\left(u^{\prime}\right)\right\rangle=$ $\left(u, u^{\prime}\right)$. The form $(-,-)$ on $U$ is positive semidefinite, and clearly $y:=\sum_{\alpha \in \Delta} c_{\alpha} e_{\alpha} \in$ $U$ spans its radical. Following [2, Ch. V, $\S 4.9$ ], let $U^{*}$ denote the dual space of $U$ and $F$ denote the affine subspace $F=\left\{f \in U^{*} \mid f(y)=1\right\}$. This is a coset of the subspace $T:=\left\{f \in U^{*} \mid f(y)=0\right\}$ of $U^{*}$, so $T$ acts simply transitively by translation on $F$. Clearly $T$ is canonically isomorphic to $(U / \mathbb{R} y)^{*}$. The form $\langle-,-\rangle$ on $U$ induces a natural positive definite inner product on the quotient $U / \mathbb{R} y$, which may thus be naturally identified with $T:=(U / \mathbb{R} y)^{*}$, which therefore has a natural induced structure of finite-dimensional real inner product space. Note that since $\left\langle y, e_{\alpha}\right\rangle=0$ for all $\alpha \in \Gamma, W_{M}$ fixes $y$ and hence acts on $F$ as a group of affine 
isometries. Let $C:=\left\{f \in U^{*} \mid f(\Delta) \subseteq \mathbb{R}_{\geq 0}\right\}$ denote the (closed) standard fundamental chamber for $\left(W_{M}, S_{M}\right)$ acting on $U^{*}$. It is a closed simplicial cone, with walls $\left\{f \in U^{*} \mid f\left(e_{\alpha}\right)=0\right\}$ for $\alpha \in \Gamma$. The group $W$ acts faithfully by restriction as a group of affine transformations of $F$, and we shall therefore identify $W$ with a group of affine transformations of $F$ (cf. [2, Ch. V, §4, Prop. 10]). Then $W$ is a faithful, effective, indecomposable group generated by affine reflections. The intersection $A:=C \cap F$ is a closed fundamental alcove for $W$ acting on $F$. Note that $A$ is a closed simplex in $F$. Its walls are the intersections of $F$ with the walls of $C$ and hence are also indexed naturally by $\Gamma$. Consider the unit normals (in $T$ ) to the walls of $C$, in the direction of $C$ from the wall. The matrix of inner products of these normals is $\left(e_{\alpha}, e_{\beta}\right)_{\alpha, \beta \in \Gamma}$ by [2, Ch. V, $\S 4$, Prop. 10].

By [2, Ch. V, $\S 3$, Prop. 12], we may choose a special point $O$ of $W_{M}$ on $F$ such that $O \in A$. Taking the origin to be $0=O$, the affine space $F$ acquires the structure of a real Euclidean space, which is mapped isomorphically to $T$ by translation by $-O$. Denote the inner product on $F$ as $(-\mid-)$. It follows from [2, Ch. VI, $\S 2$, Prop. 8] that $W_{M}$ acting on $F$ is the affine Weyl group of a reduced crystallographic essential root system $\Psi$ in $F$, as in Proposition 1, with Weyl group $W_{0}$. Since $W_{M}$ is indecomposable, $\Psi$ is indecomposable. Every closed alcove of $W_{M}$ on $F$ is contained in a closed chamber of $W_{0}$ on $F$. Choose a set of simple roots $\Lambda$ for $\Psi$ such that the corresponding closed fundamental chamber $C^{\prime}$ for $W_{0}$ contains $A$. Then $A$ is the unique alcove of $W_{M}$ in $F$ which is contained in $C^{\prime}$ and which contains 0 in its closure. Hence $A$ is the closed fundamental alcove for $\left(W_{M}, S_{M}\right)$. Let $\omega_{0}$ denote the highest root of $\Psi$ with respect to $\Lambda$. Then we have by Proposition 1 that

$$
A=\left\{v \in F \mid(v \mid \beta) \geq 0 \text { for all } \beta \in \Gamma \text { and }\left(v \mid \omega_{0}\right) \leq 1\right\} .
$$

It follows that the unit normals to the walls of $A$ are $\left\{\dot{\beta} \mid \beta \in \Lambda^{\prime}\right\}$, where $\Lambda^{\prime}:=$ $\Lambda \cup\left\{-\omega_{0}\right\}$.

This shows that the matrix $((\dot{\alpha} \mid \dot{\beta}))_{\alpha, \beta \in \Lambda^{\prime}}$ coincides (up to permutations of its

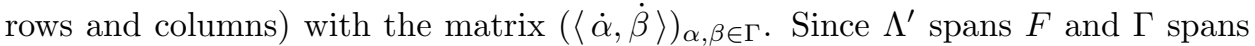
$\mathbb{R} \Gamma$, this implies that there is a unique identification of $F=\mathbb{R} \Gamma$ as inner product spaces such that $\{\dot{\alpha} \mid \alpha \in \Gamma\}=\left\{\dot{\beta} \mid \beta \in \Lambda^{\prime}\right\}$. Let $\theta \in \Gamma$ with $\dot{\theta}=\dot{\theta}_{0}$. We may write $\Lambda^{\prime}=\left\{c_{\alpha} \alpha \mid \alpha \in \Gamma\right\}$ for uniquely determined $c_{\alpha} \in \mathbb{R}_{>0}$. All assertions of the proposition are now clear.

3.9. The following corollary provides a classification-free proof of the claim at the end of the proof of Theorem 1 .

Corollary 3. Let $\Phi$ be a crystallographic root system, and let $\Gamma \subseteq \Phi$ be an indecomposable subset which is linearly dependent over $\mathbb{R}$ and satisfies $\langle\alpha, \beta\rangle \leq 0$ for all distinct $\alpha, \beta \in \Gamma$. Then

(a) Let $B:=(\langle\dot{\alpha}, \dot{\beta}\rangle)_{\alpha, \beta \in \Gamma}$ and $M=\left(m_{\alpha, \beta}\right)_{\alpha, \beta \in \Pi}$, where $m_{\alpha, \beta}$ denote the order of $s_{\alpha} s_{\beta}$ if $\alpha \neq-\beta$, and $m_{\alpha, \beta}:=\infty$ if $\alpha=-\beta$. Then $M$ is the Coxeter matrix of an indecomposable affine Weyl group, and $B=B_{M}$.

(b) Let $W^{\prime}:=\left\langle s_{\alpha} \mid \alpha \in \Gamma\right\rangle$. There is an element $\theta \in \Gamma$ such that $\Gamma^{\prime}:=\Gamma \backslash\{\theta\}$ is a simple system for the root system $\Phi^{\prime} \subseteq \Phi$ of $W^{\prime}$, and $\theta \in \Phi^{\prime}$.

Proof. Clearly $M$ is a Coxeter matrix. Since $\Phi$ is crystallographic, rank two considerations [2, Ch. VI, §1.3] imply that $\langle\dot{\alpha}, \dot{\beta}\rangle=-\cos \frac{\pi}{m_{\alpha, \beta}}$ for all $\alpha, \beta \in \Gamma$. Hence $B=B_{M}$, and thus we are in the situation of Proposition 3, It follows from this 
proposition that $M$ is the Coxeter matrix of an indecomposable affine Weyl group whose root system we denote by $\Psi$. Let $\theta$ be as in the conclusion of Proposition 3 ,

Since $\Gamma^{\prime}:=\Gamma \backslash\{\theta\}$ is linearly independent, it is a simple system of some root subsystem $\Phi^{\prime}$ of $\Phi$, by Lemma 1, and evidently $\Phi^{\prime}:=W^{\prime} \Gamma^{\prime}$, where $W^{\prime}:=\left\langle s_{\alpha}\right|$ $\left.\alpha \in \Gamma^{\prime}\right\rangle$. Moreover, since some multiple $c_{\theta} \theta \in \Phi^{\prime}$, the reflection $s_{\theta} \in W^{\prime}$ and hence $W^{\prime}=\left\langle s_{\alpha} \mid \alpha \in \Gamma\right\rangle$. It also follows that there is some $\alpha \in \Phi^{\prime}$ with $s_{\alpha}=s_{\theta}$. But $\alpha \in \Phi^{\prime}$ and $s_{\alpha}=s_{\theta}$ implies $\alpha= \pm \theta$, whence $\theta \in \Phi^{\prime}$.

\section{Subsystems of AfFine Root Systems I}

In this section, we classify the reflection subgroups of $W^{a}$ (a) up to equality and (b) up to isomorphism. The classification (b) was conjectured (in equivalent form) and partly proved by Coxeter [5] and was proved in [8]. The proof here via the stronger result (a) is along similar lines, but it is much simpler and independent of the classification of affine Weyl groups. The classification in (a) is obtained in conjunction with a classification of all simple subsystems of $\Phi^{a}$ (up to equality). The reflection subgroups are in bijective correspondence with those simple subsystems which are contained in a fixed positive subsystem of the ambient affine root system.

4.1. Let $\Phi$ be a crystallographic root system in $V$ and $\Phi^{a}$ the corresponding affine root system (in $\widehat{V}$; see Definition 4). For non-isotropic $v \in \widehat{V}$, we henceforth write $s_{v}$ for the orthogonal reflection in $v^{\perp}$, so that for $x \in \widehat{V}, s_{v}(x)=x-\left\langle x, \frac{2 v}{\langle v, v\rangle}\right\rangle v$. Note that this transformation $s_{v}$ was denoted $\widehat{s}_{v}$ in 3.3 . A root subsystem $\Sigma$ of $\Phi^{a}$ is a subset of $\Phi^{a}$ with the property that $s_{\alpha}(\beta) \in \Sigma$ for all $\alpha, \beta \in \Sigma$. The root subsystems of $\Phi^{a}$ are in natural bijective correspondence with the reflection subgroups of $W^{a}$ by the map which to a reflection subgroup $W^{\prime}$ associates the set of all roots $\alpha$ in $\Phi^{a}$ such that $s_{\alpha} \in W^{\prime}$. The inverse map associates to a root subsystem $\Sigma$ the subgroup $\left\langle s_{\alpha} \mid \alpha \in \Sigma\right\rangle$ (here we use simple consequences of properties of reflection subgroups of Coxeter systems from [8]).

The notion of a simple subsystem of $\Phi^{a}$ is more delicate than the corresponding notion for $\Phi$ (see [16, 6]). Consider the following set of real numbers:

$$
\operatorname{COS}:=\left\{-\cos \frac{\pi}{m} \mid m \in \mathbb{N}_{\geq 2}\right\} \cup(-\infty,-1] \subseteq \mathbb{R} .
$$

Let $\Sigma$ be a root subsystem of $\Phi^{a}$ with corresponding reflection subgroup $W^{\prime}$. A subset $\Gamma$ of $\Phi^{a}$ is called a simple system for $\Sigma$ if $W^{\prime}=\left\langle s_{\alpha} \mid \alpha \in \Gamma\right\rangle$, $\Gamma$ is positively independent and $\langle\dot{\alpha}, \dot{\beta}\rangle \in-\operatorname{COS}$ for all distinct $\alpha, \beta \in \Gamma$. Then $\Sigma=W^{\prime} \Gamma$.

Remark 3. In [6] the notion of an abstract simple system is defined by replacing positive independence of $\Gamma$ by the weaker condition that each component of $\Gamma$ be positively independent. In this work we shall not make use of this concept.

For any simple system $\Lambda$ of $\Sigma, S_{\Lambda}:=\left\{s_{\alpha} \mid \alpha \in \Lambda\right\}$ is a set of Coxeter generators of $W^{\prime}$. If $\Sigma \subseteq \Phi$, its simple systems in the above sense coincide with those in the sense of $\oiint 1$

Note that any subset of $\Phi_{+}^{a}$ is positively independent. It therefore follows from the general theory of reflection subgroups of Coxeter systems ([8], [9]) that $\Sigma$ has a unique simple system $\Lambda$ contained in $\Phi_{+}$; we call this the canonical simple system of $\Sigma$ (or of the corresponding reflection subgroup). We write $\Lambda=\Delta_{W^{\prime}}$. Then $S_{\Lambda}$ is the 'canonical' set of Coxeter generators of $W^{\prime}$ (in the sense of [8]), with respect to the simple reflections $S^{a}$ of $W^{a}$. If $\Sigma$ is irreducible, then any two simple systems 
of $\Sigma$ are in the same $W^{\prime} \times\{ \pm 1\}$-orbit, as follows from [24, from $\$ 3.7$ and [16, or from [6].

If $\Delta$ is a simple system of some root subsystem $\Sigma$ of $\Phi^{a}$, we refer to $\Delta$ as a simple subsystem of $\Phi^{a}$. If $\Delta \subseteq \Phi$, we write $W_{\Delta}:=\left\langle s_{\alpha} \mid \alpha \in \Delta\right\rangle$ for the subgroup of $W$ generated by reflections corresponding to elements of $\Phi$ and $\Phi_{\Delta}=W_{\Delta} \Delta:=\{\alpha \in$ $\left.\Phi \mid s_{\alpha} \in W_{\Delta}\right\}$ for the corresponding root subsystem of $\Phi$.

4.2. Let $\pi$ denote the projection $\widehat{V} \rightarrow V$ with $\delta \mapsto 0$. Note that

$$
\pi\left(\Phi^{a}\right) \subseteq \Phi, \quad\langle\alpha, \beta\rangle=\langle\pi(\alpha), \pi(\beta)\rangle \text { for } \alpha, \beta \in \Phi^{a} .
$$

The next result follows immediately from this by well-known properties [2, Ch. VI, $\S 2$, no. 3] of rank two root subsystems of $\Phi$.

Lemma 3. (a) A subset $\Delta$ of $\Phi$ (resp. $\Phi^{a}$ ) is a simple subsystem of $\Phi$ if and only if $\Delta$ is positively independent, and $\langle\alpha, \beta\rangle \leq 0$ for all distinct $\alpha, \beta \in \Delta$.

(b) A subset $\Delta$ of $\Phi_{+}$(resp. $\left.\Phi_{+}^{a}\right)$ is the canonical simple system of some reflection subgroup $W^{\prime}$ of $W$ (resp. $\left.W^{a}\right)$ if and only if $\langle\alpha, \beta\rangle \leq 0$ for all distinct $\alpha, \beta \in \Delta$.

Remark 4. More generally, this lemma applies mutatis mutandis to real root systems of Kac-Moody Lie algebras; e.g. see [24, 5.5, Theorem 1].

\subsection{Negative product subsets.}

Definition 5. We say that a subset $\Delta$ of $\widehat{V}$ is an np subset (negative product subset) if $\langle\alpha, \beta\rangle \leq 0$ for all distinct $\alpha, \beta \in \Delta$.

Lemma 4. (a) $\Delta \subseteq \Phi^{a}$ is an $n p$ subset if and only if the restriction of $\pi$ to $\Delta$ is injective and $\pi(\Delta)$ is an np subset of $\Phi$.

(b) Given an np subset $\Delta^{\prime}$ of $\Phi$, the np subsets $\Delta$ of $\Phi^{a}$ with $\pi(\Delta)=\Delta^{\prime}$ are the sets of the form $\Delta=\left\{\alpha+f(\alpha) \delta \mid \alpha \in \Delta^{\prime}\right\}$, where $f: \Delta^{\prime} \rightarrow \mathbb{Z}$ is an arbitrary function.

(c) For $\Delta$ and $f$ as in (b), We have $\Delta \subseteq \Phi_{+}^{a}$ if and only if $f(\alpha) \geq 0$ for $\alpha \in \Phi_{+}$ and $f(\alpha)>0$ for $\alpha \in-\Phi_{+}$.

Proof. This follows directly from (13).

4.4. As an immediate corollary of Lemmas 3 and 4, we obtain a parameterisation of the reflection subgroups of $W^{a}$ (or equivalently, root subsystems of $\Phi^{a}$ ) in terms of np subsets of $\Phi$.

For any additive subgroup $Z$ of $\mathbb{R}$, let $\mathcal{R}(\Phi, Z)$ denote the set of all pairs $(\Gamma, f)$ where $\Gamma$ is an np subset of $\Phi$ and $f: \Gamma \rightarrow Z \cap \mathbb{R}_{\geq 0}$ is a function such that $f(\alpha)>0$ if $\alpha \in-\Phi_{+} \cap \Gamma$.

Theorem 2. The reflection subgroups of the affine Weyl group $W^{a}$ are in natural bijective correspondence with $\mathcal{R}(\Phi, \mathbb{Z})$. The correspondence attaches to $(\Gamma, f) \in$ $\mathcal{R}(\Phi, \mathbb{Z})$ the unique reflection subgroup $W^{\prime}$ of $W^{a}$ with $\Delta_{W^{\prime}}=\{\alpha+f(\alpha) \delta: \alpha \in \Gamma\}$.

We denote the reflection subgroup $W^{\prime}$ of $W^{a}$ attached to the pair $(\Gamma, f) \in$ $\mathcal{R}(\Phi, \mathbb{Z})$ of the theorem by $W^{a}(\Gamma, f)$, its corresponding root system in $\Phi^{a}$ by $\Phi^{a}(\Gamma, f)$, and its canonical simple system by $\Delta(\Gamma, f) \subseteq \Phi_{+}$. A particular case is $W^{a}=W^{a}\left(\Gamma_{0}, f_{0}\right)$, where $\left(\Gamma_{0}, f_{0}\right) \in \mathcal{R}(\Phi, \mathbb{Z})$ is defined by $\Gamma_{0}=\pi(\widehat{\Pi})$ and

$$
f_{0}(\alpha):= \begin{cases}0, & \text { if } \alpha \in \Phi_{+}, \\ 1, & \text { if } \alpha \in-\Phi_{+} .\end{cases}
$$


4.5. Classification of $\mathbf{n p}$ subsets. The np subsets of $\Phi$ are described in the following lemma.

Lemma 5. Let $\Gamma^{\prime}$ be a simple subsystem of $\Phi$ with indecomposable components $\Gamma_{i}^{\prime}$ for $i=1, \ldots, n$. For each $i$, let $\Gamma_{i}$ denote either $\Gamma_{i}^{\prime}$ or $\Gamma_{i}^{\prime} \cup\left\{\alpha_{i}\right\}$, where either $-\alpha_{i}$ is the highest root of $\Phi_{\Gamma_{i}^{\prime}}$ or $-\alpha_{i}^{\vee}$ is the highest root of $\Phi_{\Gamma_{i}^{\prime}}^{\vee}$ (or both). Then $\Gamma:=\bigcup_{i} \Gamma_{i}$ is an np subset of $\Phi$ whose components are the $\Gamma_{i}$. Moreover, any np subset $\Gamma$ of $\Phi$ is obtained in this way from some simple subsystem $\Gamma^{\prime}$ of $\Phi$.

Proof. Well-known properties of highest roots [2, Ch. VI,§1. 8] imply that $\Gamma$ as in the lemma is an np subset of $\Phi$. The converse follows by applying Proposition 3 to the indecomposable components $\Gamma_{i}$ of $\Gamma$ and using Lemma 2 .

Remark 5. Suppose $\Gamma$ is an np subset of $\Phi$. The components $\Gamma_{i}$ are uniquely determined by $\Gamma$, but $\Gamma_{i}$ need not uniquely determine $\alpha_{i}$ and $\Gamma_{i}^{\prime}$ as above if $\Gamma_{i}^{\prime} \neq \Gamma_{i}$. However, $W_{\Gamma}=W_{\Gamma^{\prime}}$, and any two choices of $\Gamma^{\prime}$ (for fixed $\Gamma$ ) are in the same $W_{\Gamma}$ orbit. See also Remark 13.

4.6. We now obtain the following known description ([8] of the reflection subgroups of $W^{a}$, up to isomorphism as Coxeter systems.

Theorem 3. Consider a root subsystem $\Psi$ of $\Phi$ with indecomposable components $\Psi_{i}$ for $i=1, \ldots, n$. For each $i$, let $W_{i}$ denote either the Weyl group of $\Psi_{i}$, the affine Weyl group corresponding to $\Psi_{i}$, or the affine Weyl group corresponding to $\Psi_{i}^{\vee}$. Each $W_{i}$ has a standard realisation as a Coxeter system, with simple reflections determined up to conjugacy.

The affine Weyl group $W^{a}$ has a reflection subgroup isomorphic to $W^{\prime}:=W_{1} \times$ $\ldots \times W_{n}$ as a Coxeter system. Conversely, any reflection subgroup of $W^{a}$ is isomorphic to a Coxeter system $W^{\prime}$ arising in this way.

Proof. Using Lemma 5, the isomorphism type of the reflection subgroup attached to $(\Gamma, f)$ in Theorem 2 may be described as follows. Suppose $\Gamma$ is obtained as in Lemma 5 from the simple subsystem $\Gamma^{\prime}$ of $\Phi$, and let $\Gamma_{i}, \Gamma_{i}^{\prime}$ be as the sets of roots defined there. Then $W^{\prime}$ has indecomposable components $W_{i}^{\prime}$ with simple roots $\Delta_{i}=\left\{\alpha+f(\alpha) \delta: \alpha \in \Gamma_{i}\right\}$, for $i=1, \ldots, n$. If $\Gamma_{i}=\Gamma_{i}^{\prime}$, then $W_{i}^{\prime}$ is isomorphic to the Weyl group of $\Phi_{\Gamma_{i}^{\prime}}$. If $\Gamma_{i}=\Gamma_{i}^{\prime} \cup\left\{\alpha_{i}\right\}$, where $-\alpha_{i}$ is the highest root of $\Phi_{\Gamma_{i}^{\prime}}$, then $W_{i}^{\prime}$ is isomorphic to the affine Weyl group of $\Phi_{\Gamma_{i}^{\prime}}$. Finally, if $\Gamma_{i}=\Gamma_{i}^{\prime} \cup\left\{\alpha_{i}\right\}$, where $-\alpha_{i}^{\vee}$ is the highest root of $\Phi_{\Gamma_{i}^{\prime}}^{\vee}$, then $W_{i}^{\prime}$ is isomorphic to the affine Weyl group of $\Phi_{\Gamma_{i}^{\prime}}^{\vee}$

4.7. The next lemma gives necessary and sufficient conditions for an np subset $\Delta \subseteq \Phi^{a}$ to be positively independent in terms of its parameterisation in Lemma 4(b).

Lemma 6. Let $\Gamma$ be an $n p$ subset of $\Phi$ and $f: \Gamma \rightarrow \mathbb{Z}$ be a function. Let $\Gamma_{i}$ for $i=1, \ldots, n$ denote the components of $\Gamma$. Let $J$ denote the set of indices $i$ with $1 \leq i \leq n$ and $\Gamma_{i}$ linearly dependent. For each $i \in J$ there are unique integers $c_{\gamma} \in \mathbb{N}_{>0}$ for $\gamma \in \Gamma_{i}$ such that $\sum_{\gamma \in \Gamma_{i}} c_{\gamma} \gamma=0$ and $\operatorname{gcd}\left(\left\{c_{\gamma} \mid \gamma \in \Gamma_{i}\right\}\right)=1$; we set $d_{i}:=\sum_{\gamma \in \Gamma_{i}} c_{\gamma} f_{\gamma}$. Let $\Delta:=\{\gamma+f(\gamma) \delta \mid \gamma \in \Gamma\}$. Then:

(a) $\Delta$ is positively independent if and only if either $d_{i}>0$ for all $i \in J$ or $d_{i}<0$ for all $i \in J$. 
(b) Each component of $\Delta$ is positively independent if and only if $d_{i} \neq 0$ for all $i \in J$.

Proof. Each $\Gamma_{i}$ is either linearly independent or there is a (real) linear relation among its elements, which is unique up to a scalar by [2, Ch. V, §3, Lemma 5]. Since $\Gamma$ is contained in the lattice $\mathbb{Z} \Phi$, the coefficients of these linear relations can be taken in $\mathbb{Q}_{>0}$ by loc. cit., and then by rescaling, the existence and uniqueness of the $c_{\gamma}$ for $\gamma \in \Gamma_{i}, i \in J$, satisfying the conditions of the lemma follows.

For any set of scalars $a_{\gamma} \in \mathbb{R}_{\geq 0}(\gamma \in \Gamma)$, we have $\sum_{\gamma \in \Gamma} a_{\gamma}(\gamma+f(\gamma) \delta)=0$ if and only if $\sum_{\gamma} a_{\gamma} \gamma=0$ and $\sum_{\gamma} a_{\gamma} f(\gamma)=0$. Now by [2], $\sum_{\gamma} a_{\gamma} \gamma=0$ if and only if there are non-negative scalars $b_{i}$ for $i \in J$, such that $a_{\gamma}=b_{i} c_{\gamma}$ if $\gamma \in \Gamma_{i}$ for some $i \in J$, and $a_{\gamma}=0$ otherwise. For such scalars $a_{\gamma}$, we have $\sum_{\gamma} a_{\gamma} f(\gamma)=\sum_{i \in J} b_{i} d_{i}$. Hence $\Delta$ is positively independent precisely if, for all non-negative scalars $b_{i}(i \in J)$ which are not all zero, $\sum_{i \in J} b_{i} d_{i} \neq 0$. The assertion (a) follows immediately from this. The components of $\Delta$ are $\Delta_{i}:=\left\{\alpha+f(\alpha) \delta: \alpha \in \Gamma_{i}\right\}$ for $i=1, \ldots, n$, so (b) follows from (a) applied to each $\Gamma_{i}$.

Definition 6. If the condition in (a) (resp. (b)) holds, we say that $(\Gamma, f)$ is compatible (resp. strongly compatible).

4.8. The following parameterisation of simple subsystems of $\Phi^{a}$ follows immediately from Lemma [6] and the definitions, in the same way as Theorem 2.

Proposition 4. The simple subsystems of the root system $\Phi^{a}$ of $W^{a}$ are in natural bijective correspondence with pairs $(\Gamma, f)$, where $\Gamma$ is an np subset of $\Phi$ and $f: \Gamma \rightarrow$ $\mathbb{Z}$ is a function such that $(\Gamma, f)$ is compatible. The correspondence attaches to $(\Gamma, f)$ the simple subsystem $\{\alpha+f(\alpha) \delta: \alpha \in \Gamma\}$ of $\Phi^{a}$.

Remark 6. We note that rank 1 simple subsystems of $\Phi^{a}$ correspond naturally to roots in $\Phi^{a}$. Theorem 2 and Proposition 4 directly generalise the descriptions ((5) and Proposition 2) of the positive roots and roots of $\Phi^{a}$ in terms of those of $\Phi$.

4.9. Short and long roots. For any non-isotropic $v \in \widehat{V}$, let $k_{v}=\langle v, v\rangle \in \mathbb{R}_{>0}$. If $\Psi$ is a subset of some indecomposable crystallographic root system in $V$, then $\left\{k_{v} \mid v \in \Psi\right\}$ has either 1 or 2 elements. If this set has two elements, we denote them by $k_{\Psi,+}$ and $k_{\Psi,-}$ where $k_{\Psi,+}>k_{\Psi,-}$. Otherwise, we define $k_{\Psi,+}=k_{\Psi,-}:=k_{v}$ for any $v \in \Psi$. Set $k_{\Psi}:=\frac{k_{\Psi,+}}{k_{\Psi,-}}$. It is known from [2] that $k_{\Psi} \in\{1,2,3\}$. Write $\Psi_{\text {short }}:=\left\{v \in \Psi \mid\langle v, v\rangle=k_{\Psi,-}\right\}$ and $\Psi_{\text {long }}:=\left\{v \in \Psi \mid\langle v, v\rangle=k_{\Psi,+}\right\}$.

Using the above notation and that of Lemma [5] we now define $k_{\beta, \Gamma} \in\{1,2,3\}$ for any np subset $\Gamma$ and element $\beta \in \Gamma$. There is a unique component $\Gamma_{i}$ in which $\beta$ lies. If $\Gamma_{i}=\Gamma_{i}^{\prime}$, we set $k_{\beta, \Gamma}:=1$. Otherwise, $\Gamma_{i} \neq \Gamma_{i}^{\prime}$, and we set

$$
k_{\beta, \Gamma}:= \begin{cases}1, & \text { if } k_{\alpha_{i}}=k_{\Gamma_{i},+}, \\ \frac{k_{\beta}}{k_{\alpha_{i}}} \in\left\{1, k_{\Gamma_{i}}\right\}, & \text { if } k_{\alpha_{i}}=k_{\Gamma_{i},-} .\end{cases}
$$

4.10. The technical lemma below describes the root coefficients in certain root subsystems of $\Phi^{a}$. Let $\Gamma$ be an indecomposable np subset of $\Phi$. Let $\Gamma^{\prime}:=\Gamma$ if $\Gamma$ is linearly independent. Otherwise, write $\Gamma=\Gamma^{\prime} \cup\{-\alpha\}$, where $\Gamma^{\prime}$ is an indecomposable simple subsystem of $\Phi$ and $\left\langle\alpha, \Gamma^{\prime}\right\rangle \subseteq \mathbb{R}_{\geq 0}$. Let $\Sigma$ be the root subsystem of $\Phi$ with simple system $\Gamma^{\prime}$. 
Lemma 7. Maintain the above notation. If $\Gamma=\Gamma^{\prime}$, set $\Delta^{\prime}:=\Gamma$; otherwise, set $\Delta^{\prime}:=\Gamma^{\prime} \cup\{\omega\} \subseteq \Phi^{a}$, where $\omega:=\delta-\alpha$. Let $W^{\prime}:=\left\langle s_{\gamma} \mid \gamma \in \Delta^{\prime}\right\rangle$. Then $W^{\prime}$ is a reflection subgroup of $W^{a}$ having $\Delta^{\prime}$ as a simple system. Let $\Psi^{\prime}:=W^{\prime} \Delta$ denote the indecomposable root subsystem of $\Phi^{a}$ corresponding to $\Delta^{\prime}$. Then:

(a) Define coefficients $a_{\beta, \gamma}$ for $\beta \in \Sigma$ and $\gamma \in \Gamma^{\prime}$ by $\beta=\sum_{\gamma \in \Gamma^{\prime}} a_{\beta, \gamma} \gamma$. If $\Gamma$ is linearly dependent, then

$$
\beta+n \delta=n \omega+\sum_{\gamma \in \Gamma^{\prime}}\left(a_{\beta, \gamma}+n a_{\alpha, \gamma}\right) \gamma
$$

for $\beta \in \Sigma$ and $n \in \mathbb{Z}$.

(b) If $\Gamma$ is linearly independent, then $\Psi^{\prime}=\Sigma$.

(c) If $\Gamma$ is linearly dependent, then $\Psi^{\prime}=\left\{\beta+n k_{\beta, \Gamma} \delta \mid \beta \in \Sigma, n \in \mathbb{Z}\right\}$.

Proof. Parts (a) and (b) are trivial. In (c), the case $\Gamma \neq \Gamma^{\prime}$ with $k_{\alpha}=k_{\Gamma,+}$ is a special case of Proposition 2 since then all $k_{\beta, \Gamma}=1$. The remaining case is that in which $\Gamma \neq \Gamma^{\prime}$ and $k_{\alpha}=k_{\Gamma,-}$. Set $b_{\beta}=\frac{2}{k_{\beta}}$ for $\beta \in \Sigma$, so $\beta^{\vee}=b_{\beta} \beta$. We have $k_{\alpha^{\vee}}=k_{\Gamma^{\vee},+}$. Consider $\Delta^{\prime \vee}=\Gamma^{\vee \vee} \cup\left\{b_{\alpha} \delta-\alpha^{\vee}\right\}$. Here, $\delta^{\prime}:=b_{\alpha} \delta$ plays the analogous role for $\Delta^{\prime \vee}$ to that played by $\delta$ for $\Delta^{\prime}$. By the case of (c) previously considered, the root system $\Psi^{\prime \vee}$ corresponding to $\Delta^{\prime \vee}$ is

$$
\Psi^{\prime \vee}=\left\{\beta+n \delta^{\prime} \mid \beta \in \Sigma^{\vee}, n \in \mathbb{Z}\right\}=\left\{\beta^{\vee}+n b_{\alpha} \delta \mid \beta \in \Sigma, n \in \mathbb{Z}\right\} .
$$

Hence

$$
\Psi^{\prime}=\left\{\left(\beta^{\vee}+n b_{\alpha} \delta\right)^{\vee} \mid \beta \in \Sigma, n \in \mathbb{Z}\right\}=\left\{\beta+n \frac{b_{\alpha}}{b_{\beta}} \delta \mid \beta \in \Sigma, n \in \mathbb{Z}\right\},
$$

which is as required since $\frac{b_{\alpha}}{b_{\beta}}=\frac{k_{\beta}}{k_{\alpha}}=k_{\beta, \Gamma}$.

4.11. Let $\Gamma$ be an np subset of $\Phi$ and let $f: \Gamma \rightarrow \mathbb{Z}$ such that $(\Gamma, f)$ is compatible. We assume that $\Gamma$ arises as in Lemma 5 from a simple system $\Gamma^{\prime} \subseteq \Gamma$ and use the notation $\Gamma_{i}, \Gamma_{i}^{\prime}, \alpha_{i}$ introduced there.

For each $i$ with $\Gamma_{i}^{\prime} \neq \Gamma_{i}$, there is a unique linear relation $\sum_{\gamma \in \Gamma_{i}} c_{\gamma} \gamma=0$ with $c_{\alpha_{i}}=1$, and in this relation, we have $c_{\gamma} \in \mathbb{N}_{>0}$ for all $\alpha \in \Gamma_{i}$.

Let $\Sigma=\Phi_{\Gamma^{\prime}}$ and $\Sigma_{i}=\Phi_{\Gamma_{i}^{\prime}}$. Thus $\Sigma$ is a root system with indecomposable components $\Sigma_{i}$, of which $\Gamma^{\prime}$ is a simple system, and $\Gamma^{\prime} \subseteq \Gamma \subseteq \Sigma$. As in Lemma 7 . for each $\beta \in \Sigma$, write $\beta=\sum_{\gamma \in \Gamma^{\prime}} a_{\beta, \gamma} \gamma$. Then $a_{\beta, \gamma} \in \mathbb{Z}, a_{\beta, \gamma}=0$ unless $\beta \in \Sigma_{i}$ and $\gamma \in \Gamma_{i}^{\prime}$ for some $i$, and for fixed $\beta \in \Sigma$, either all $a_{\beta, \gamma}$ are non-negative or they are all non-positive.

By Proposition 4, $\Delta:=\{\gamma+f(\gamma) \delta \mid \gamma \in \Gamma\}$ is a simple subsystem of $\Phi^{a}$. Let $W^{\prime \prime}:=\left\langle s_{\gamma} \mid \gamma \in \Delta\right\rangle$ be the associated Coxeter group, and let $\Psi:=W^{\prime \prime} \Delta$ be the corresponding root system.

Define $r_{\beta} \in \mathbb{Z}$ for all $\beta \in \Sigma$ by $r_{\beta}:=\sum_{\gamma \in \Gamma^{\prime}} a_{\beta, \gamma} f(\gamma)=-r_{-\beta}$. We also define $K_{i} \in \mathbb{Z}$ for $i=1, \ldots, n$ as follows. If $\Gamma_{i}=\Gamma_{i}^{\prime}$, we set $K_{i}=0$. Otherwise, set $K_{i}:=\sum_{\gamma \in \Gamma_{i}} c_{\gamma} f(\gamma)=f\left(\alpha_{i}\right)+r_{-\alpha_{i}}$.

Proposition 5. Let $\Gamma$ be an np subset of $\Phi$ and let $f: \Gamma \rightarrow \mathbb{Z}$ be a function such that $(\Gamma, f)$ is compatible. Then the root system $\Psi$ of which $\Delta:=\{\alpha+f(\alpha) \delta \mid \alpha \in \Gamma\}$ is a simple system given by

$$
\Psi=\left\{\beta+\left(r_{\beta}+m K_{i} k_{\beta, \Gamma}\right) \delta \mid i=1, \ldots, n, \beta \in \Sigma_{i}, m \in \mathbb{Z}\right\} .
$$


Remark 7. The above result applies in particular in the case where $\Delta \subseteq \Phi_{+}^{a}$ when $\Psi=\Phi^{a}(\Gamma, f)$. In that case, we have $r_{\beta} \geq 0$ for all $\beta \in \Sigma$ which are positive with respect to $\Gamma^{\prime}$. If, further, $\Gamma_{i}^{\prime} \neq \Gamma_{i}$, then using [2, Ch. VI, §1, Prop. 25] for $\Sigma$ and $\Sigma^{\vee}$, we find that $0 \leq r_{\beta} \leq K_{i} k_{\beta, \Gamma}$ for all $\beta \in \Sigma_{i}$ which are positive with respect to $\Gamma_{i}^{\prime}$.

Proof. The proof easily reduces to the case where $\Gamma$ is indecomposable, which we henceforth assume. Let $\Delta^{\prime}, \Psi^{\prime}$ be as in Lemma 7. There is an isometry $i: \mathbb{R} \Delta^{\prime} \rightarrow$ $\mathbb{R} \Delta$, mapping the basis $\Delta^{\prime}$ of the left side to the basis $\Delta$ of the right side. More precisely, $\gamma \mapsto \gamma+f(\gamma) \delta$ for $\gamma \in \Gamma^{\prime}$, and, if $\Gamma^{\prime} \neq \Gamma, \delta-\alpha \mapsto f(-\alpha) \delta-\alpha$. Clearly, this isometry induces an isomorphism $W^{\prime} \rightarrow W^{\prime \prime}$ of Coxeter systems and maps $\Psi^{\prime}$ bijectively to $\Psi$. From the expressions for elements of $\Psi^{\prime}$ as linear combinations of the simple roots $\Delta^{\prime}$ in Lemma 7, we obtain corresponding expressions for elements of $\Psi$ as linear combinations of simple roots $\Delta$. One readily checks that expanding the latter linear combinations gives the desired result, using the definitions of $r_{\beta}$, $K_{i}$ and $k_{\beta, \Gamma}$.

4.12. Weyl data, alcoves and compatible pairs. For the remainder of this section, fix $(\Gamma, f) \in \mathcal{R}(\Phi, \mathbb{Z})$. We shall show how to explicitly describe in terms of the data $(\Gamma, f)$ the fundamental alcove of $W^{\prime}:=W^{a}(\Gamma, f)$, the volume of an alcove, the index $\left[W^{a}: W^{\prime}\right]$, and the minimal length (say, left) coset representatives of $W^{\prime}$ in $W^{a}$. To describe these results, we maintain all the notation from the last subsection.

It is clear that $\Phi^{a}(\Gamma, f)$ is finite (equivalently, $W^{a}(\Gamma, f)$ is finite) if and only if $\Gamma=\Gamma^{\prime}$. Also, $W^{a}(\Gamma, f)$ has only affine type components (i.e. no finite components) if and only if $\Gamma_{i} \neq \Gamma_{i}^{\prime}$ for all $i$. Finally, $W^{a}(\Gamma, f)$ is of finite index in $W^{a}$ if and only if its translation subgroup is of finite index in that of $W^{a}$, which is the case if and only if $\Gamma_{i} \neq \Gamma_{i}^{\prime}$ for all $i$ and $\mathbb{R} \Phi=\mathbb{R} \Gamma^{\prime}$.

4.13. The fundamental coweights $\omega_{\alpha}:=\omega_{\alpha}\left(\Gamma^{\prime}\right)$ for $\alpha \in \Gamma^{\prime}$ are defined as the basis of $\mathbb{R} \Gamma^{\prime}$ which is dual to $\Gamma^{\prime}$ with respect to $\langle-,-\rangle$. Recall that $f_{\Gamma^{\prime}}$ denotes the index of connection of $\Phi_{\Gamma^{\prime}}\left(=\left[\sum_{\gamma \in \Gamma^{\prime}} \mathbb{Z} \omega_{\gamma}: \sum_{\gamma \in \Gamma^{\prime}} \mathbb{Z} \gamma\right]\right)$ and let $r_{i}:=\left|\Gamma_{i}^{\prime}\right|$ denote the rank of $\Phi_{\Gamma_{i}^{\prime}}$.

Lemma 8. (a) The closed fundamental alcove for $W^{a}(\Gamma, f)$ on $V$ is

$$
C=C(\Gamma, f):=\{v \in V \mid\langle v, \gamma\rangle+f(\gamma) \geq 0 \text { for all } \gamma \in \Gamma\}
$$

(b) $C=\perp_{i=0}^{n} C_{i}$ (the orthogonal direct sum of subsets), where $C_{0}$ is the subspace $C_{0}:=\{v \in V \mid\langle v, \Gamma\rangle=0\}$, and for $i=1, \ldots, n$,

$$
C_{i}=\left\{v \in \mathbb{R} \Gamma_{i}^{\prime} \mid\langle v, \gamma\rangle+f(\gamma) \geq 0 \text { for all } \gamma \in \Gamma_{i}\right\} .
$$

(c) For $i=1, \ldots, n$ let $v_{i, 0}:=-\sum_{\alpha \in \Gamma_{i}^{\prime}} f(\alpha) \omega_{\alpha}$. If $\Gamma_{i}^{\prime}=\Gamma_{i}$, then $C_{i}$ is the simplicial cone $v_{i, 0}+\sum_{\alpha \in \Gamma_{i}} \mathbb{R}_{\geq 0} \omega_{\alpha}$ with vertex $v_{i_{0}}$, while if $\Gamma_{i}^{\prime} \neq \Gamma_{i}$, then $C_{i}$ is the simplex with vertices $v_{i, 0}$ and $v_{i, 0}+\frac{K_{i}}{c_{\alpha}} \omega_{\alpha}$ for $\alpha \in \Gamma_{i}^{\prime}$.

Proof. We refer to [20, Proposition 3.12], 2, Ch. V, §4, no. 4-6], [24, 5.6] and [9] for basic facts concerning fundamental chambers and Tits cones used below. The closed fundamental chamber for $W^{a}(\Gamma, f)$ acting on $\widehat{V}^{*}$ is

$$
\left.\widehat{C}:=\left\{\phi \in \widehat{V}^{*} \mid \phi(\alpha+f(\alpha) \delta)\right) \geq 0 \text { for all } \alpha \in \Gamma\right\},
$$


and the corresponding Tits cone is

$$
\widehat{X}:=\left\{\phi \in \widehat{V}^{*} \mid \phi(\beta)<0 \text { for at most finitely many } \beta \in \Phi^{a}(\Gamma, f) \cap \Phi_{+}\right\} .
$$

Clearly, $\widehat{X}$ contains the Tits cone of $W^{a}$ acting on $\widehat{V}^{*}$, which is

$$
\left\{\phi \in \widehat{V}^{*} \mid \phi(\beta)<0 \text { for at most finitely many } \beta \in \Phi_{+}^{a}\right\} \supseteq i(V),
$$

where $i: V \rightarrow \widehat{V}^{*}$ is as in the proof of Proposition 2 Since $\widehat{C}$ is a fundamental domain for $W^{a}(\Gamma, f)$ on $\widehat{X}$ and $i(V)$ is $W$-stable, it follows that $C$ is a fundamental domain for $W^{a}(\Gamma, f)$ acting on $V$. The reflections in the walls of $C$ correspond to the reflections in the walls of $\widehat{C}$, which are the canonical Coxeter generators of $W^{a}(\Gamma, f)$, and (a) follows.

Part (b) follows readily from (a) and the definitions.

For (c), note first that $C_{i}$ is a simplicial cone or simplex, depending on whether $\Gamma_{i}^{\prime}=\Gamma_{i}$ or $\Gamma_{i}^{\prime} \neq \Gamma_{i}$. In either case, one verifies first that $v_{i, 0}$ lies on all walls of $C_{i}$ of the form $\left\langle v \in \mathbb{R} \Gamma_{i} \mid\langle v, \gamma\rangle+f(\gamma)=0\right\rangle$ for $\gamma \in \Gamma_{i}^{\prime}$. If $\Gamma_{i}^{\prime}=\Gamma_{i}$, then the elements $\omega_{\alpha}$ are parallel to the extreme rays of $C_{i}$ and the assertion of (c) holds. If $\Gamma_{i}^{\prime} \neq \Gamma_{i}$, then one checks that

$$
\left\langle v_{i, 0}, \alpha_{i}\right\rangle+f\left(\alpha_{i}\right)=K_{i}
$$

so that $v_{i_{0}} \in C_{i}$ since $K_{i}>0$. Then for $\alpha \in \Gamma_{i}^{\prime}$, one sees from (15) that the point $v_{i, 0}+\frac{K_{i}}{c_{\alpha}} \omega_{\alpha}$ lies on all faces of $C_{i}$ except the face $\left\langle v \in \mathbb{R} \Gamma_{i} \mid\langle v, \alpha\rangle+f(\alpha)=0\right\rangle$, so it is a vertex of $C_{i}$. The result (c) follows.

4.14. Volume. We maintain the notation of the above section and, in addition, consider Lebesgue measure $\mu$ on $V$, normalised so that the measure of an $m$ dimensional hypercube in $V$ with sides of unit length (using the metric arising from $\langle-,-\rangle)$ is 1 , where $m=\operatorname{dim}(V)$. Recall that $(\Gamma, f)$ is a fixed element of $\mathcal{R}(\Phi, \mathbb{Z})$, which defines a root subsystem of $\Phi^{a}$ as in Proposition 4 . By Lemma 8 the chamber $C$ is a fundamental domain for the action of $W\left(\Phi^{a}(\Gamma, f)\right)$ on $V$.

Corollary 4. (a) If the measure $\mu(C)$ is finite, then

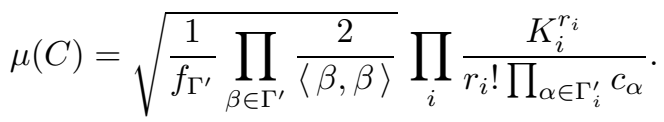

(b) $\mu(C)$ is finite if and only if the index $\left[W^{a}: W^{a}(\Gamma, f)\right]$ is finite.

(c) Suppose also that $\left(\Gamma^{\prime}, f^{\prime}\right) \in \mathcal{R}(\Phi, \mathbb{Z})$ with $W^{a}(\Gamma, f) \subseteq W^{a}\left(\Gamma^{\prime}, f^{\prime}\right)$ and that both $\mu(C(\gamma, f))$ and $\mu\left(C\left(\Gamma^{\prime}, f^{\prime}\right)\right)$ are finite. Then

$$
\left[W^{a}\left(\Gamma^{\prime}, f^{\prime}\right): W^{a}(\Gamma, f)\right]=\frac{\mu(C(\Gamma, f))}{\mu\left(C\left(\Gamma^{\prime}, f^{\prime}\right)\right)} .
$$

Remark 8. Since $W^{a}=W^{a}\left(\Gamma_{0}, f_{0}\right)$, the measure $\mu(A)$ of the fundamental alcove of $W^{a}$ is finite and may be computed as a special case of the formula in (a).

Proof. If the measure $\mu(C)$ is finite, then $\mathbb{R} \Phi=\mathbb{R} \Gamma$ and for all $i, \Gamma_{i}^{\prime} \neq \Gamma_{i}$. We assume we are in this situation. Let $\mu_{i}$ denote Lebesgue measure on $\mathbb{R} \Gamma_{i}$, normalised in a similar way to $\mu$. We have $\mu(C)=\prod_{i} \mu\left(C_{i}\right)$ since the $C_{i}$ are pairwise orthogonal. Consider the full-dimensional parallelepiped $P_{i}$ in $\mathbb{R} \Gamma_{i}$ with 0 as one vertex and with the edges incident with 0 given by endpoints $\omega_{\alpha}$ for $\alpha \in \Gamma_{i}^{\prime}$. By the change of variables formula in multiple integrals, its volume is

$$
\mu_{i}\left(P_{i}\right)=\sqrt{\operatorname{det}\left(\left\langle\omega_{\alpha}, \omega_{\beta}\right\rangle\right)_{\alpha, \beta \in \Gamma_{i}^{\prime}}}
$$


(this is also a well known formula of Hadamard). By expressing the elements of $\Gamma^{\prime}$ and the corresponding coroots and fundamental coweights in terms of an orthonormal basis of $\mathbb{R} \Gamma_{i}$ and by using (3), one checks that the determinant under the square root sign is equal to

$$
\frac{1}{f_{\Gamma_{i}^{\prime}}} \prod_{\beta \in \Gamma_{i}^{\prime}} \frac{2}{\langle\beta, \beta\rangle} .
$$

On the other hand, it is easy to see using Lemma 8 (c) and the change of variable formula that

$$
\mu_{i}\left(C_{i}\right)=\mu_{i}\left(P_{i}\right) \prod_{i} \frac{K_{i}^{n_{i}}}{n_{i} ! \prod_{\alpha \in \Gamma_{i}^{\prime}} c_{\alpha}} .
$$

Combining the above facts, using $f_{\Gamma^{\prime}}=\prod_{i} f_{\Gamma_{i}^{\prime}}$, gives (a). Parts (b) and (c) follow readily from [2, Ch. VI, §2, Lemma 1].

4.15. From [8], the set $\left\{w \in W^{a} \mid w \Delta(\Gamma, f) \subseteq \Phi_{+}^{a}\right\}$ is known to be the set of minimal length left coset representatives of $W^{a}(\Gamma, f)$ in $W^{a}$ (where length is with respect to the standard length function of $\left.\left(W^{a}, S^{a}\right)\right)$. The next result explicitly describes a set of left coset representatives of $W^{a}(\Gamma, f)$ in $\widetilde{W}=W \ltimes \tau_{R}$ (with $R$ as in $(3.4)$, reducing to the above set if $\widetilde{W}=W^{a}$, i.e. if $R=Q$.

Proposition 6. Let $G:=\left\{w \in \widetilde{W} \mid w \Delta(\Gamma, f) \subseteq \Phi_{+}^{a}\right\}$. Then:

(a) For $w \in W$ and $\gamma \in R$, we have $w t_{\gamma} \in G$ if and only if for all $\alpha \in \Gamma$,

$$
\begin{cases}\langle\alpha, \gamma\rangle \geq-f(\alpha), & \text { if } w(\alpha) \in \Phi_{+}, \\ \langle\alpha, \gamma\rangle \geq 1-f(\alpha), & \text { if } w(\alpha) \in \Phi_{-} .\end{cases}
$$

(b) For $x \in \widetilde{W}$, we have $x \in G$ if and only if $x^{-1}(A) \subseteq C$. Further, we have $C=\bigcup_{x \in G} x^{-1}(A)$

(c) $G \cong \widetilde{W} / W^{a}(\Gamma, f)$ is a set of left coset representatives of $W^{a}(\Gamma, f)$ in $\widetilde{W}$.

(d) $|G|=\left[W^{a}: W^{a}(\Gamma, f)\right] \cdot[R: Q]$.

Proof. Part (a) follows easily from the definition of $\Phi^{a}(\Gamma, f)$ and (10). Note that $W^{a}$ acts simply transitively on the set of its alcoves and $\widetilde{W}=W \ltimes \tau_{R}$ acts on the set of alcoves. It follows that $\widetilde{W}$ is the semidirect product of its normal subgroup $W^{a}$ with the subgroup

$$
\{w \in \widetilde{W} \mid w(A)=A\}=\{w \in \widetilde{W} \mid w(\widetilde{\Pi})=\widetilde{\Pi}\} \cong R / Q,
$$

and this reduces the proofs of (b)-(d) to the case $\widetilde{W}=W^{a}$ where the statements are standard or trivial.

\section{Subsystems of AfFine Root systems II}

In the previous section, root subsystems of $\Phi^{a}$ were classified using the concept of a negative product (np) set, which arises naturally in considering simple subsystems of affine root systems. In this section we give an alternative classification in terms of collections of subsets of $\mathbb{Z}$. 
5.1. For subsets $A, B$ of any abelian group, define $A \pm B:=\{a \pm b \mid a \in A, b \in B\}$ and $n A=\{n a \mid a \in A\}$ for $n \in \mathbb{Z}$. Note that $2 A \neq A+A$ in general.

Any subset $\Psi$ of $\Phi^{a}$ may be described as $\Psi:=\left\{\alpha+n \delta \mid \alpha \in \Phi, n \in Z_{\alpha}\right\}$, where, for each $\alpha \in \Phi, Z_{\alpha}$ is a subset of $\mathbb{Z}$. Given subsets $Z_{\alpha}$ for $\alpha \in \Phi$, the next lemma gives necessary and sufficient conditions on the $Z_{\alpha}$ in order that the corresponding set $\Psi$ be a root subsystem of $\Phi^{a}$.

Lemma 9. Let $\left\{Z_{\alpha} \mid \alpha \in \Phi\right\}$ be a family of (possibly empty) subsets of $\mathbb{Z}$. Then $\Psi:=\left\{\alpha+n \delta \mid \alpha \in \Phi, n \in Z_{\alpha}\right\}$ is a root subsystem of $\Phi^{a}$ if and only if, for all $\alpha, \beta \in \Phi$, we have

$$
Z_{\beta}-\left\langle\beta, \alpha^{\vee}\right\rangle Z_{\alpha} \subseteq Z_{s_{\alpha}(\beta)}
$$

Proof. This follows directly from (9) and the definition of a root subsystem.

5.2. The root subsystems of $\Phi^{a}$ may be described in terms of solutions of (16) as follows.

Corollary 5. The root subsystems of $\Phi^{a}$ are in bijective correspondence with pairs $\left(\Psi,\left\{Z_{\alpha}\right\}_{\alpha \in \Psi}\right)$, where $\Psi$ is a root subsystem of $\Phi$ and $\left\{Z_{\alpha}\right\}_{\alpha \in \Psi}$ is a family of nonempty subsets of $\mathbb{Z}$ satisfying (16) for all $\alpha, \beta \in \Psi$. The correspondence attaches to $\left(\Psi,\left\{Z_{\alpha}\right\}_{\alpha \in \Psi}\right)$ the root subsystem $\Phi^{a}\left(\Psi,\left\{Z_{\alpha}\right\}_{\alpha \in \Psi}\right):=\left\{\alpha+n \delta \mid \alpha \in \Psi, n \in Z_{\alpha}\right\}$ of $\Phi^{a}$.

Proof. Observe that for any solution $\left\{Z_{\alpha}\right\}_{\alpha \in \Phi}$ of (16), $\left\{\alpha \in \Phi \mid Z_{\alpha} \neq \emptyset\right\}$ is clearly a root subsystem $\Psi$ of $\Phi$; hence the corresponding root subsystem of $\Phi^{a}$ is of the required form. Conversely, if $\Psi$ is a subsystem of $\Phi$ and $\left\{Z_{\alpha}\right\}_{\alpha \in \Psi}$ satisfy (16) for all $\alpha, \beta \in \Psi$, a solution of (16) is obtained by taking $Z_{\alpha}=\emptyset$ for $\alpha \in \Phi \backslash \Psi$. The corresponding root subsystem of $\Phi^{a}$ is then $\Phi^{a}\left(\Psi,\left\{Z_{\alpha}\right\}_{\alpha \in \Psi}\right)$.

Note that under the above bijection, the root subsystems of $\Phi$ correspond to the case where each non-empty subset $\mathbb{Z}_{\alpha}=\{0\}$.

5.3. The above description of root subsystems is convenient for studying the inclusion relations in the poset of root subsystems of $\Phi^{a}$. In fact, it is obvious that $\Phi^{a}\left(\Psi,\left\{Z_{\alpha}\right\}_{\alpha \in \Psi}\right) \subseteq \Phi^{a}\left(\Psi^{\prime},\left\{Z_{\alpha}^{\prime}\right\}_{\alpha \in \Psi}\right) \Longleftrightarrow \Psi \subseteq \Psi^{\prime}$ and $Z_{\alpha} \subseteq Z_{\alpha}^{\prime}$ for all $\alpha \in \Psi^{\prime}$.

The next lemma describes the natural action of the extended affine Weyl group on the root subsystems of $\Phi^{a}$. This corresponds to the conjugation action of $\widetilde{W}^{a}$ on the set of reflection subgroups of its normal subgroup $W^{a}$. The action is described for reflections in $W$ and for translations, which together generate $\widetilde{W}^{a}$.

Lemma 10. Let $w \in \widetilde{W}^{a}$ and write $w\left(\Phi^{a}\left(\Psi,\left\{Z_{\alpha}\right\}_{\alpha \in \Psi}\right)\right)=\Phi\left(\Psi^{\prime},\left\{Z_{\alpha}^{\prime}\right\}_{\alpha \in \Psi^{\prime}}\right)$.

(a) If $w \in W$, then $\Psi^{\prime}=w(\Psi)$ and $Z_{\alpha}^{\prime}=Z_{w^{-1}(\alpha)}$ for $\alpha \in \Psi^{\prime}$.

(b) If $w=t_{\gamma}$ where $\gamma \in P(\Phi)$ (the coweight lattice), then $\Psi^{\prime}=\Psi$ and $Z_{\alpha}^{\prime}=$ $Z_{\alpha}+\langle\alpha, \gamma\rangle$ for all $\alpha \in \Psi^{\prime}$.

Proof. This follows directly from the definitions and (10).

5.4. For any indecomposable subset $\Gamma$ of $\Phi$, define another subset $\Gamma^{\circ}$ of $V$ as follows:

$$
\Gamma^{\circ}=\left\{\alpha \mid \alpha \in \Gamma_{\text {short }}\right\} \cup\left\{\frac{1}{k_{\Gamma}} \alpha \mid \alpha \in \Gamma_{\text {long }}\right\}
$$

(see $\$ 4.9$ for the notation). If $\Psi$ is an indecomposable root system in $V$ with simple system $\Gamma$, then $\Psi^{\circ}$ is a root system in $V$ (homothetic to $\Psi^{\vee}$, and hence isomorphic to $\Psi^{\vee}$ ) with simple system $\Gamma^{\circ}$, and $W_{\Psi}=W_{\Psi^{\circ}}$. 
5.5. Fix an additive subgroup $Z$ of $\mathbb{R}$. Let $\Psi$ be a root subsystem of $\Phi$, with components $\Psi_{1} \ldots \Psi_{n}$.

A subgroup $X$ of $P_{Z}(\Psi)$ is said to be a $Z$-admissible coweight lattice for $\Psi$ if it is of the form $X=\bigoplus_{i=1}^{n} X_{i} \subseteq P(\Psi)$, where either $X_{i}=0$, in which case we set $m_{i}=0$, or $X_{i}=m_{i} P\left(\Psi_{i}\right)$ or $X_{i}=m_{i} P\left(\Psi_{i}^{\circ}\right)$ for some $m_{i} \in Z \cap \mathbb{R}_{>0}$. The $Z$-admissible coroot lattice (for $\Psi$ ) corresponding to $X$ is then defined to be the group $Y=\sum_{i} Y_{i}$, where $Y_{i}=0$ if $X_{i}=0$, and $Y_{i}=m_{i} Q\left(\Psi_{i}\right)\left(\right.$ resp., $\left.Y_{i}=m_{i} Q\left(\Psi_{i}^{\circ}\right)\right)$ if $X_{i}=P\left(\Psi_{i}\right)$ (resp., $X_{i}=P\left(\Psi_{i}^{\circ}\right)$ ). This gives a natural bijective correspondence between $Z$-admissible coweight lattices and $Z$-admissible coroot lattices of $\Psi$.

We take $Z=\mathbb{Z}$ here. We shall require some facts about the elements $n_{\alpha} \in \mathbb{N}$ defined by $\langle\alpha, X\rangle=n_{\alpha} \mathbb{Z}$ for all $\alpha \in \Psi$. Choose a simple system $\Delta_{i}$ for $\Psi_{i}$ and set $\Delta:=\bigcup_{i} \Delta_{i}$. If $\alpha \in \Delta$, write $\omega_{\alpha}=\omega_{\alpha}(\Delta)$ for the corresponding fundamental coweight. We have $X=\bigoplus_{i} \bigoplus_{\alpha \in \Delta_{i}} n_{\alpha}^{\prime} \mathbb{Z} \omega_{\alpha}$, where for $\alpha \in \Delta_{i}$ we set $n_{\alpha}^{\prime}=m_{i} k_{\Psi_{i}}$ if $X_{i}=m_{i} P\left(\Psi_{i}{ }^{\circ}\right)$ and $\alpha$ is long in $\Psi_{i}$, and otherwise set $n_{\alpha}^{\prime}=m_{i}$. It follows that if $\alpha \in \Delta$, we have $n_{\alpha}=n_{\alpha}^{\prime}$. Since $n_{\alpha}=n_{w(\alpha)}$ for $w \in W_{\Psi}$, by the $W_{\Psi \text {-invariance }}$ of $P\left(\Psi_{i}\right)$ and $P\left(\Psi_{i}^{\circ}\right)$, it follows that

$$
n_{\alpha}=m_{i} k_{i, \alpha}
$$

for all $\alpha \in \Psi_{i}$, where $k_{i, \alpha}=k_{\Psi_{i}}$ if $\alpha$ is long in $\Psi_{i}$ and $X_{i}=m_{i} P\left(\Psi_{i}^{\circ}\right)$, and $k_{i, \alpha}=1$ otherwise.

It also follows that

$$
X=\left\{p \in P(\Psi) \mid\langle p, \alpha\rangle \subseteq n_{\alpha} \mathbb{Z} \text { for all } \alpha \in \Psi\right\} .
$$

To see this, note that the left hand side is clearly contained in the right. Moreover, if an element $x=\sum_{\alpha \in \Delta} a_{\alpha} \omega_{\alpha}$ of $P(\Psi)$ is in the right hand side, then for $\alpha \in \Delta_{i}$ we have $a_{\alpha}=\langle x, \alpha\rangle \in n_{\alpha} \mathbb{Z}=n_{\alpha^{\prime}} \mathbb{Z}$, and so $x \in X$. Another consequence of (17) which we shall use is the divisibility property

$$
\left\langle\beta, \alpha^{\vee}\right\rangle n_{\alpha} \mathbb{Z} \subseteq n_{\beta} \mathbb{Z}
$$

for $\alpha, \beta \in \Psi$. This is trivial unless $\alpha, \beta$ are in the same component $\Psi_{i}$, are not orthogonal, $X_{i}=m_{i} P\left(\Psi_{i}{ }^{\circ}\right)$ and $\beta$ is long and $\alpha$ is short in $\Psi_{i}$; but then $\left\langle\beta, \alpha^{\vee}\right\rangle=$ $k_{\Psi}$ by rank two considerations, and the result still holds.

5.6. Second parameterisation. The next theorem gives our second main parameterisation of the root subsystems of $\Phi^{a}$. To formulate it, let $\mathcal{R}^{\prime}(\Phi, Z)$ denote the set of all pairs $(\Psi, X)$, where $\Psi$ is a root subsystem of $\Phi$ and $X \in P_{Z}(\Psi) / X^{\prime}$ is a coset $X=a+X^{\prime}$, where $a \in P_{Z}(\Psi)$, of some $Z$-admissible coweight lattice $X^{\prime}$ of $\Psi$.

Theorem 4. There is a natural bijection between $\mathcal{R}^{\prime}(\Phi, \mathbb{Z})$ and the set of root subsystems of $\Phi^{a}$. The bijection attaches to $(\Psi, X) \in \mathcal{R}^{\prime}(\Phi, \mathbb{Z})$ the root subsystem $\Phi^{a}(\Psi, X):=\Phi^{a}\left(\Psi,\left\{Z_{\alpha}\right\}_{\alpha \in \Psi}\right)$, where $Z_{\alpha}:=\{\langle\alpha, x\rangle \mid x \in X\} \subseteq \mathbb{Z}$ and $\Phi^{a}\left(\Psi,\left\{Z_{\alpha}\right\}_{\alpha \in \Psi}\right):=\left\{\alpha+n \delta \mid \alpha \in \Psi, n \in Z_{\alpha}\right\}$.

Proof. First we show that there is a map $g:(\Psi, X) \mapsto \Phi^{a}(\Psi, X)$ as indicated from $\mathcal{R}^{\prime}(\Phi, \mathbb{Z})$ to the set of root subsystems of $\Phi^{a}$. Fix $(\Psi, X) \in \mathcal{R}^{\prime}(\Phi, \mathbb{Z})$ and define $Z_{\alpha}:=\langle\alpha, X\rangle \subseteq \mathbb{Z}$. for $\alpha \in \Psi$. Write $X=a+X^{\prime}$, where $X^{\prime}$ is an admissible coweight lattice for $\Psi$ and $a \in P(\Psi)$. Note that $Z_{\alpha}=\langle a, \alpha\rangle+\mathbb{Z} n_{\alpha}$ for the unique integer $n_{\alpha} \geq 0$, such that $n_{\alpha} \mathbb{Z}=\left\langle X^{\prime}, \alpha\right\rangle$. It is easy to check from (17) and (19) that the pair $\left(\Psi,\left\{Z_{\alpha}\right\}_{\alpha \in \Psi}\right)$ satisfies the condition of Corollary 5 , and so determines a root subsystem $g(\Psi, X)=\Phi^{a}\left(\Psi,\left\{Z_{\alpha}\right\}_{\alpha \in \Psi}\right)$ as in Corollary 5 . 
We now show that $g$ is injective. Fix $(\Psi, X) \in \mathcal{R}^{\prime}(\Phi, \mathbb{Z})$ and define $Z_{\alpha}:=$ $\langle\alpha, X\rangle \subseteq \mathbb{Z}$ for $\alpha \in \Psi$ as above. Since

$$
\Psi=\left\{\alpha \in \Phi \mid \alpha+n \delta \in \Phi^{a}(\Psi, X) \text { for some } n \in \mathbb{Z}\right\},
$$

it will suffice to show that $\left\{Z_{\alpha}\right\}_{\alpha \in \Psi}$ uniquely determines $(\Psi, X)$. It follows from (18) that

$$
X^{\prime}=\left\{p \in P(\Psi) \mid\langle p, \alpha\rangle \subseteq n_{\alpha} \mathbb{Z} \text { for all } \alpha \in \Psi\right\} .
$$

From this, we obtain $X=\left\{p \in P(\Psi) \mid\langle p, \alpha\rangle \subseteq Z_{\alpha}\right.$ for all $\left.\alpha \in \Psi\right\}$. The injectivity of the map $g$ follows.

Now to show that $g$ is surjective, it will suffice to show that for $(\Gamma, f)$ as in Theorem 2, there is some pair $(\Psi, X)$ with $\Phi^{a}(\Gamma, f)=\Phi^{a}(\Psi, X)$. In the following argument, we use the notation of 4.11 concerning $\Gamma$. Choose $\Psi=\Sigma$ and $p \in P(\Sigma)$ such that $\langle p, \alpha\rangle=r_{\alpha}$ for all $\alpha \in \Gamma^{\prime}$ and define $X^{\prime}=\bigoplus_{i} X_{i} \subseteq P(\Sigma)$ where

$$
X_{i}^{\prime}:= \begin{cases}0, & \text { if } \Gamma^{\prime}=\Gamma_{i}, \\ K_{i} P\left(\Gamma_{i}\right), & \text { if } \Gamma^{\prime} \neq \Gamma_{i} \text { and } \alpha_{i, 0} \text { is long, } \\ K_{i} P\left(\Gamma_{i}{ }^{\circ}\right), & \text { if } \Gamma^{\prime} \neq \Gamma_{i} \text { and } \alpha_{i, 0} \text { is short. }\end{cases}
$$

Then $X^{\prime}=\bigoplus X_{i}^{\prime}$ is a $\mathbb{Z}$-admissible coweight lattice of $P(\Sigma)$, and from Proposition 5 and (17) we see that $\Phi^{a}\left(\Sigma, p+X^{\prime}\right)=\Phi^{a}(\Gamma, f)$. This completes the proof.

5.7. Evidently there is a unique bijection $j=j_{\mathbb{Z}}: \mathcal{R}(\Phi, \mathbb{Z}) \rightarrow \mathcal{R}^{\prime}(\Phi, \mathbb{Z})$ such that, setting $j(\Gamma, f)=(\Psi, X)$, we have $\Phi^{a}(\Psi, X)=\Phi^{a}(\Gamma, f)$. The above proof indicates how to compute $j(\Gamma, f)$ explicitly from $(\Gamma, f)$. Computing $j^{-1}(\Psi, X)$ amounts to determining the canonical simple system of $\Phi^{a}(\Psi, X)$. We describe below one simple way in which this may be done; a more natural procedure using alcove geometry will be described in 96 .

Suppose $(\Psi, X) \in \mathcal{R}^{\prime}(\Phi, \mathbb{Z})$. We may find $(\Gamma, f) \in \mathcal{R}(\Phi, \mathbb{Z})$ with $\Phi^{a}(\Gamma, f)=$ $\Phi^{a}(\Psi, X)$ as follows. For $\alpha \in \Psi$, write $Z_{\alpha}=\langle X, \alpha\rangle \subseteq \mathbb{Z}$ and $Z_{\alpha}^{\prime}:=\left\{n \in Z_{\alpha} \mid\right.$ $\left.\alpha+n \delta \in \Phi_{+}^{a}\right\}$. Let $\Psi^{\prime}:=\left\{\alpha \in \Psi \mid Z_{\alpha}^{\prime} \neq \emptyset\right\}$, and for $\alpha \in \Psi^{\prime}$, let $r_{\alpha}^{\prime}:=\min \left(Z_{\alpha}^{\prime}\right)$. Let $\Delta$ denote the simple system contained in $\Phi_{+}^{a}$ of $\Phi^{a}(\Psi, X)$ and $\Delta^{\prime \prime}:=\left\{\alpha+r_{\alpha}^{\prime} \alpha \mid\right.$ $\left.\alpha \in \Psi^{\prime}\right\}$. One then checks that $\Delta \subseteq \Delta^{\prime \prime}$. It is well known that $\Delta$ is the unique inclusion-minimal subset $\Delta^{\prime}$ of $\Phi_{+}^{a} \cap \Phi^{a}(\Psi, X)$ such that $\Phi^{a}(\Psi, X) \cap \Phi_{+}^{a} \subseteq \mathbb{N} \Delta^{\prime}$. It follows that $\Delta$ is the unique inclusion-minimal subset $\Delta^{\prime}$ of the finite set $\Delta^{\prime \prime}$ such that $\Delta^{\prime \prime} \subseteq \mathbb{N} \Delta^{\prime}$. Thus, $\Delta$ in $\Phi_{+}^{a}$ may be effectively determined. Set $\Gamma=\pi(\Delta)$ (an np subset in $\Phi)$ and define $f: \Gamma \rightarrow \mathbb{Z}_{\geq 0}$ by $f(\alpha)=r_{\alpha}^{\prime}$. Then $\Phi^{a}(\Gamma, f)=\Phi^{a}(\Psi, X)$.

5.8. We may now use the action of $\widetilde{W}^{a}$ on $V$ to describe the $\widetilde{W}^{a}$-action on root subsystems of $\Phi^{a}$ in terms of their parameterisation in Theorem 4 . For any subset $\Sigma$ of $\Phi$, we let $p_{\Sigma}: V \rightarrow \mathbb{R} \Sigma$ denote the orthogonal projection of $V$ on $\mathbb{R} \Sigma$.

Corollary 6. Let $w=t_{\gamma} x \in \widetilde{W}^{a}$, where $x \in W$ and $\gamma \in P(\Phi)$. Let $(\Psi, X) \in$ $\mathcal{R}^{\prime}(\Phi, \mathbb{Z})$. Then $\Psi^{\prime}:=x(\Psi)$ is a root subsystem of $\Phi$ and $X^{\prime \prime}:=p\left(\tau_{\gamma} x(X)\right)$ is a coset in $P\left(\Psi^{\prime}\right)$ of some admissible coweight lattice of $P\left(\Psi^{\prime}\right)$, where $p=p_{\Psi^{\prime}}$. Further, $w \Phi^{a}(\Psi, X)=\Phi^{a}\left(\Psi^{\prime}, X^{\prime \prime}\right)$.

Remark 9. Note that the isomorphism $t_{\gamma} x \leftrightarrow \tau_{\gamma} x$ between the affine and linear versions of $\widetilde{W}^{a}$ used implicitly above is different from the one $\left(t_{\gamma} x \leftrightarrow \tau_{-\gamma} x\right)$ used in Proposition 2 
Proof. Write $X=a+X^{\prime}$, where $a \in P(\Psi)$ and $X^{\prime}$ is an admissible coweight lattice of $P(\Psi)$. Clearly, $\Psi^{\prime}$ is a root subsystem of $\Phi$ and $X^{\prime \prime \prime}:=x\left(X^{\prime}\right)$ is an admissible coweight lattice of $P\left(\Psi^{\prime}\right)$. We have $\tau_{\gamma} x(X)=\gamma+x(a)+X^{\prime \prime \prime}$, where clearly $x(a) \in P\left(\Psi^{\prime}\right)$. Hence $p \tau_{\gamma} x(X)=p(\gamma)+x(a)+X^{\prime \prime}$. To prove the assertion about $X^{\prime \prime}$, it will suffice to show that $p(\gamma) \in P\left(\Psi^{\prime}\right)$. But this holds since for any $\alpha \in \Psi^{\prime}$ we have $\langle\alpha, p(\gamma)\rangle=\langle\alpha, \gamma\rangle \in \mathbb{Z}$. This proves the first claim. The final claim follows from Lemma 10 as follows. Write $Z_{\alpha}=\langle X, \alpha\rangle$ for $\alpha \in \Psi$. Then by Lemma 10, $w \Phi^{a}(\Psi, X)=w \Phi^{a}\left(\Psi,\left\{Z_{\alpha}\right\}_{\alpha \in \Psi}\right)=\Phi^{a}\left(\Psi^{\prime},\left\{Z_{\alpha}^{\prime}\right\}_{\alpha \in \Psi^{\prime}}\right)$, where for $\alpha \in \Psi^{\prime}, Z_{\alpha}^{\prime}=Z_{x^{-1} \alpha}+\langle\alpha, \gamma\rangle=\left\langle x^{-1} \alpha, X\right\rangle+\langle\alpha, \gamma\rangle$. But from the above, $X^{\prime \prime}=p \tau_{\gamma} x(X)=p(\gamma)+x(a)+x\left(X^{\prime}\right)=p(\gamma)+x(X)$, and so

$$
\left\langle\alpha, X^{\prime \prime}\right\rangle=\langle\alpha, p(\gamma)+x(X)\rangle=\langle\alpha, p(\gamma)\rangle+\langle\alpha, x(X)\rangle=\langle\alpha, \gamma\rangle+\left\langle x^{-1} \alpha, X\right\rangle .
$$

Hence $\Phi^{a}\left(\Psi^{\prime},\left\{Z_{\alpha}^{\prime}\right\}_{\alpha \in \Psi^{\prime}}\right)=\Phi^{a}\left(\Psi^{\prime}, X^{\prime}\right)$, as required.

5.9. Affine versus finite. Now fix $(\Psi, X) \in \mathcal{R}^{\prime}(\Phi, \mathbb{Z})$ and write $X=b+X^{\prime}$, where $b \in P(\Psi)$ and $X^{\prime}$ is an admissible coweight lattice of $P(\Psi)$. Let $\left\{\Psi_{i}\right\}$ be the components of $\Psi$, and write $X^{\prime}=\sum_{i} X_{i}^{\prime}$, where for each $i$ either $X_{i}^{\prime}=m_{i} P\left(\Psi_{i}\right)$, $X_{i}^{\prime}=m_{i} P\left(\Psi_{i}^{\circ}\right)$ with $m_{i} \in \mathbb{Z}_{>0}$, or $X_{i}^{\prime}=0$ and $m_{i}:=0$. Set $Y_{i}:=m_{i} Q\left(\Psi_{i}\right)$, $Y_{i}:=m_{i} Q\left(\Psi_{i}^{\circ}\right)$, or $Y_{i}:=0$ accordingly, and define $Y=\sum_{i} Y_{i}$. It is clear that $\Phi^{a}(\Psi, X)$ is finite (equivalently, $W^{a}(\Psi, X)$ is finite) if and only if $X^{\prime}=\{0\}$. Also, $W^{a}(\Psi, X)$ has only affine type components (i.e. no finite components) if and only if $X_{i}^{\prime} \neq\{0\}$ for all $i$. Finally, $W^{a}(\Psi, X)$ is of finite index in $W^{a}$ if and only if its translation subgroup is of finite index in that of $W^{a}$, which is the case if and only if $X_{i}^{\prime} \neq 0$ for all $i$ and $\mathbb{R} \Phi=\mathbb{R} \Psi$.

5.10. We now describe a decomposition of $W^{a}(\Psi, X)$ as the semidirect product of its translation subgroup and a finite reflection group, and we explicitly describe its elements.

Proposition 7. Let $X=b+X^{\prime}$ be a coset in $P(\Psi)$ of an admissible coweight lattice $X^{\prime}$ for $\Psi$, and let $Y$ be the admissible coroot lattice corresponding to $X^{\prime}$. Then:

(a) $W^{a}(\Psi, X)$ is a semidirect product $W^{a}(\Psi, X)=W^{\prime \prime} \ltimes t_{Y}$ of the finite reflection subgroup $W^{\prime \prime}:=\left\{t_{b-w(b)} w \mid w \in W_{\Psi}\right\}$ by the translation group $t_{Y}:=\left\{t_{\gamma} \mid \gamma \in Y\right\}$.

(b) $W^{a}(\Psi, X)=\left\{t_{b-w(b)+\gamma} w \mid w \in W_{\Psi}, \gamma \in Y\right\}$.

Proof. A typical root of $\Phi^{a}(\Psi, X)$ is of the form $\alpha+\langle\alpha, b+z\rangle \delta$ where $\alpha \in \Psi$ and $z \in X^{\prime}$, with corresponding reflection $s_{\alpha+\langle\alpha, b+z\rangle \delta}=t_{\langle\alpha, b+z\rangle \alpha^{\vee}} s_{\alpha}$. For each $\alpha \in \Psi$, we have $s_{\alpha}^{\prime}:=s_{\alpha+\langle\alpha, b\rangle \delta}=t_{\langle\alpha, b\rangle \alpha^{\vee}} s_{\alpha}=t_{b-s_{\alpha} b} s_{\alpha} \in W^{a}(\Psi, X)$. It is easy to check that the reflection subgroup of $W^{a}$ generated by $s_{\alpha}^{\prime}$ for $\alpha \in \Psi$ is $W^{\prime \prime}$. For any affine Weyl group, the group of translations is generated by the translations which are products of two reflections. Therefore, the subgroup $T^{\prime}$ of translations of $W^{a}(\Psi, X)$ is generated by elements $s_{\alpha+\langle\alpha, b+z\rangle \delta} s_{\alpha+\langle\alpha, b\rangle \delta}=t_{\langle\alpha, z\rangle \alpha^{\vee}}$ for $z \in X^{\prime}$ and $\alpha \in \Psi$. Write $\left\langle\alpha, X^{\prime}\right\rangle=n_{\alpha} \mathbb{Z}$, so $T^{\prime}$ is generated by $t_{n_{\alpha} \alpha^{\vee}}$ for $\alpha \in \Psi$. But it follows easily from (17) that $T^{\prime}=t_{Y}$. The rest of (a) follows readily, and (b) follows immediately from (a).

5.11. The next result describes the pointwise stabiliser in $\widetilde{W}$ of a root subsystem and the centraliser in $\widetilde{W}$ of the corresponding reflection subgroup, where $\widetilde{W}$ is as in 3.4 . 
Proposition 8. (a) The pointwise stabiliser of $\Phi^{a}(\Psi, X)$ in $\widetilde{W}$ is the semidirect product $W^{\prime \prime} \rtimes T$, where $W^{\prime \prime}$ is the parabolic subgroup $W^{\prime \prime}=W_{\Phi \cap \Psi \perp}$ of $W$ and $T$ is the group of translations $T=t_{R \cap \Psi^{\perp}}:=\left\{t_{\gamma} \mid \gamma \in R \cap \Psi^{\perp}\right\}$.

(b) For $w \in W$ and $\gamma \in R$, wt $t_{\gamma}$ centralises $W^{a}(\Psi, X)$ if and only if for each component $\Psi_{i}$ of $\Psi$, one of the two conditions (i) or (ii) below hold:

(i) for all $\alpha \in \Psi_{i}, w(\alpha)=\alpha$ and $\langle\gamma, \alpha\rangle=0$,

(ii) $X_{i}=0$, and for all $\alpha \in \Psi_{i}, w(\alpha)=-\alpha$ and $\langle\gamma, \alpha\rangle=-2\langle a, \alpha\rangle$, where $a$ is a representative of the coset $X$.

Proof. Part (a) follows from (10), using the fact that the centraliser in $W$ of any subset $L$ of $V$ is the parabolic subgroup of $W$ generated by $s_{\alpha}$ for $\alpha \in \Phi \cap L^{\perp}$. For part (b), one notes first that $x \in \widetilde{W}$ centralises $W^{a}(\Psi, X)$ if and only if for each $\beta \in \Phi^{a}(\Psi, X)$, we have $x \beta= \pm \beta$. It is readily verified that this suffices to conclude that $w t_{\gamma}$ is in the centraliser.

To prove necessity in (b), one notes first that if $x \in \widetilde{W}$ centralises $W^{a}(\Psi, X)$, then for each $\beta \in \Phi^{a}(\Psi, X)$, we have $x \beta= \pm \beta$. Writing $x=w t_{\gamma}$ and $\beta=\alpha+n \delta$, (10) implies that $w \alpha= \pm \alpha$ for each $\alpha \in \Psi$. By irreducibility, for each component $\Psi_{i}$ of $\Psi$, we then have either $w \alpha=\alpha$ for all $\alpha \in \Psi_{i}$ or $w \alpha=-\alpha$ for all $\alpha \in \Psi_{i}$. A further application of (10) now shows that either (i) or (ii) holds.

5.12. We describe the $\widetilde{W}$-orbit of $\Phi^{a}(\Psi, X)$ and the setwise stabiliser of $\Phi^{a}(\Psi, X)$ in $\widetilde{W}$ (which coincides with the normaliser in $\widetilde{W}$ of $W^{a}(\Psi, X)$ ). For this, we consider a second root system $\Phi^{a}\left(\Psi_{1}, X_{1}\right)$, for which we use similar notation as for $\Phi^{a}(\Psi, X)$. In particular, write $X=a+X^{\prime}$, where $a$ is a representative of $X$ and $X_{1}=a_{1}+X_{1}^{\prime}$, etc.).

Proposition 9. Let $w \in W$ and $\gamma \in R$. Then:

(a) $t_{\gamma} w\left(\Phi^{a}\left(\Psi_{1}, X_{1}\right)=\Phi^{a}(\Psi, X)\right.$ if and only if $w\left(\Psi_{1}\right)=\Psi, w\left(X_{1}^{\prime}\right)=X^{\prime}$ and $a \in w\left(a_{1}\right)+p_{\Psi}(\gamma)+X^{\prime}$

(b) $\Phi^{a}\left(\Psi_{1}, X_{1}\right)$ and $\Phi^{a}(\Psi, X)$ are in the same $\widetilde{W}$-orbit if there exists $w^{\prime} \in W$ with $w^{\prime}\left(\Psi_{1}\right)=\Psi, w^{\prime}\left(X_{1}^{\prime}\right)=X^{\prime}$ and $a-w^{\prime}\left(a_{1}\right) \in p_{\Psi}\left(R^{\prime}\right)+X^{\prime}$.

(c) $t_{\gamma} w$ normalises $W^{a}(\Psi, X)$ if and only if $w(\Psi)=\Psi, w\left(X^{\prime}\right)=X^{\prime}$ and $p_{\Psi}(\gamma) \in a-w(a)+X^{\prime}$.

Proof. Part (a) follows from Corollary [6, while (b) and (c) follow readily from (a). We omit the details.

\section{Comparison of the parameterisations}

6.1. Consider the bijection (discussed in $\left.\oint_{5.7}\right) j=j_{\mathbb{Z}}: \mathcal{R}(\Phi, \mathbb{Z}) \rightarrow \mathcal{R}^{\prime}(\Phi, \mathbb{Z}$ ), where both sides are parameter sets for the set of reflection subgroups of $W^{a}$. We indicate below how $j$ arises as the restriction (to "integer points") of a bijection $j_{\mathbb{R}}: \mathcal{R}(\Phi, \mathbb{R}) \rightarrow \mathcal{R}^{\prime}(\Phi, \mathbb{R})$, where both sides correspond naturally to the discrete reflection subgroups of the semidirect product $W \ltimes \tau_{V}$.

Let $\vec{\Phi}:=\Phi+\mathbb{R} \delta$ and

$$
\vec{\Phi}_{+}:=\left\{\alpha+n \delta \mid \alpha \in \Phi, n \in \mathbb{R}_{\geq 0}, n>0 \text { if } \alpha \in-\Phi_{+}\right\} .
$$

The group $\vec{W}^{a}:=\left\langle s_{\alpha} \mid \alpha \in \vec{\Phi}\right\rangle$ is a (generally non-discrete) group of linear transformations of $\widehat{V}$. Note that $\vec{W}^{a}$ is naturally identified as the group of affine transformations of $V$ generated by $W$ and the group of translations $\tau_{V}=\left\{\tau_{v} \mid v \in\right.$ $V\}$ of $V$. Under this identification, $\vec{W}^{a}$ is a semidirect product $\vec{W}^{a}=W \ltimes \tau_{V}$. 
We define root subsystems of $\vec{\Phi}^{a}$ as for $\Phi^{a}$. A root subsystem $\Psi$ of $\vec{\Phi}^{a}$ is discrete (as a subset of $\widehat{V}$ in the usual topology) if and only if for each $\alpha \in \Phi$, the set $Z_{\alpha}:=\{n \in \mathbb{R} \mid \alpha+n \delta \in \Psi\}$ is a discrete subset of $\mathbb{R}$. It is easy to see that this holds precisely when the corresponding reflection subgroup $\left\langle s_{\alpha} \mid \alpha \in \Psi\right\rangle$ is a discrete subgroup of $\operatorname{GL}(\widehat{V})$. It is then a Coxeter group with finite and affine components acting on $\widehat{V}$ in a similar manner to the reflection subgroups of $W^{a}$. In the affine picture, the corresponding reflection groups acting on $V$ are precisely those all of whose reflecting hyperplanes are parallel to reflecting hyperplanes of $W$.

Simple subsystems of $\vec{\Phi}^{a}$ are defined as positively independent np subsets in $\vec{\Phi}_{+}$. There are natural bijective correspondences between discrete root subsystems of $\vec{\Phi}^{a}$, discrete reflection subgroups of $\vec{W}$, and simple subsystems of $\vec{\Phi}$ which are contained in $\vec{\Phi}_{+}$. The simple subsystem of $\vec{\Phi}$ corresponding to a discrete reflection subgroup $W^{\prime}$ of $\vec{W}$ and contained in $\vec{\Phi}_{+}$will be written $\Delta_{W^{\prime}}$.

6.2. The main results (Theorems 2 and 4) hold in this more general context, with essentially the same proofs, but modified as follows: one replaces "reflection subgroups of the affine Weyl group $W^{a}$ " by "discrete reflection subgroups of $\vec{W}^{a}$," "root subsystems of $\Phi^{a}$ " by "discrete root subsystems of $\vec{\Phi}^{a}$ ", " $Z=\mathbb{Z}$ " by " $Z=\mathbb{R}$ " (and correspondingly " $\mathcal{R}(\Phi, \mathbb{Z})$ " by " $\mathcal{R}(\Phi, \mathbb{R})$ ", etc.) and " $\Phi^{a}$ " by $\vec{\Phi}$ (and correspondingly " $\Phi^{a}(\Gamma, f)$ " by $\vec{\Phi}^{a}(\Gamma, f)$ ", etc. $)$.

This gives a natural bijection $j_{\mathbb{R}}: \mathcal{R}(\Phi, \mathbb{R}) \rightarrow \mathcal{R}^{\prime}(\Phi, \mathbb{R})$ which extends $j=j_{\mathbb{Z}}$, since both sides are naturally in bijection with discrete root subsystems of $\vec{\Phi}^{a}$. The bijection $j_{\mathbb{R}}$ has similar properties to those of $j_{\mathbb{Z}}$ as discussed in $\$ 5.7$.

Remark 10. In fact, most of the theory (with obvious exceptions such as Corollary

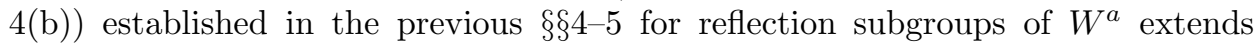
mutatis mutandis to discrete reflection subgroups of $\vec{W}^{a}$.

6.3. Let $k \subseteq \mathbb{R}$ denote either $\mathbb{Z}$ or $\mathbb{R}$. For a root subsystem $\Psi$ of $\Phi$ and a $k$-admissible subgroup $X^{\prime}=\sum_{i} X_{i}^{\prime}$ of $P_{k}(\Psi)$, we define

$$
\mathcal{R}_{\left(\Psi, X^{\prime}\right)}^{\prime}(\Phi, k):=\left\{(\Psi, X) \in \mathcal{R}^{\prime}(\Phi, k) \mid X \in P_{k}(\Psi) / X^{\prime}\right\}
$$

and $\mathcal{R}_{\left(\Psi, X^{\prime}\right)}(\Phi, k):=j_{k}^{-1}\left(\mathcal{R}_{\left(\Psi, X^{\prime}\right)}^{\prime}(\Phi, k)\right)$. Clearly, $j_{k}$ restricts to a bijection

$$
j_{k, \Psi, X^{\prime}}: \mathcal{R}_{\left(\Psi, X^{\prime}\right)}(\Phi, k) \rightarrow \mathcal{R}_{\left(\Psi, X^{\prime}\right)}^{\prime}(\Phi, k) .
$$

We have $\mathcal{R}^{\prime}(\Phi, k)=\dot{\bigcup}_{\left(\Psi, X^{\prime}\right)} \mathcal{R}_{\left(\Psi, X^{\prime}\right)}^{\prime}(\Phi, k)$ (and similarly with $\mathcal{R}^{\prime}$ replaced by $\mathcal{R}$ ), where the union is over pairs $\left(\Psi, X^{\prime}\right)$ such that $\Psi$ is a root subsystem of $\Phi$ and $X^{\prime}$ is a $k$-admissible coweight lattice in $P_{\mathbb{R}}(\Psi)$.

A preliminary discussion of the bijections $j_{\mathbb{Z}}^{ \pm 1}$ is given in 95.7 . There the description of $j_{\mathbb{Z}}$ is reasonably natural and explicit. Here we wish to provide a more natural interpretation of $j_{\mathbb{Z}}^{-1}$ than was previously available. Now $j_{\mathbb{Z}}^{-1}$ is obtained by restriction of $j_{\mathbb{R}}^{-1}$, so it is sufficient to describe the maps $j_{\mathbb{R}, \Psi, X^{\prime}}^{-1}$. The main result of this section is Theorem [5, which expresses $j_{\mathbb{R}, \Psi, X^{\prime}}^{-1}$ as a composite of maps which have natural interpretations in terms of the alcove geometry of a certain affine Weyl group $W^{\prime}=W_{\Psi, X^{\prime}}^{\prime}$ attached to $\left(\Psi, X^{\prime}\right)$. 
6.4. Fix a root subsystem $\Psi$ of $\Phi$, whose components are written as $\Psi_{i}$. We have a corresponding $\mathbb{R}$-admissible subgroup $X^{\prime}=\sum_{i} X_{i}^{\prime}$ of $P_{\mathbb{R}}(\Psi)$, where for each $i$, either $X_{i}^{\prime}=0$ and $m_{i}:=0$ or $X_{i}^{\prime}:=m_{i} P\left(\Psi_{i}\right)$ or $X_{i}^{\prime}:=m_{i} P\left(\Psi_{i}^{\circ}\right)$, where $m_{i} \in \mathbb{R}_{>0}$. Define $k_{i, \alpha}$ as above (cf. after (17) ). Let $Y^{\prime}$ be the $\mathbb{R}$-admissible coroot lattice of $\Psi$ corresponding to $X^{\prime}$, and let $W^{\prime}=W_{\Psi, X}^{\prime}$ be the group of affine transformations of $V$ generated by $W_{\Psi} \cup \tau_{Y^{\prime}}$. Note that $W^{\prime}$ acts faithfully on $\mathbb{R} \Psi$ as a discrete affine reflection group by restriction; we shall think of $W^{\prime}$ realised in this way. It is isomorphic as a Coxeter group to $W^{a}(\Psi, X)$ for any $X \in P_{\mathbb{R}}(\Psi) / X^{\prime}$.

Remark 11. For fixed $\Psi$ and varying $X$, the subgroups $W_{\Psi, X}^{\prime}$ are precisely the discrete reflection groups acting in $\mathbb{R} \Psi$ whose linear parts are (the restriction to $\mathbb{R} \Psi$ of) $W_{\Psi}$ and which have 0 as a special point.

6.5. Cosets and a fundamental domain. Denote by $\Gamma_{0}^{\prime}$ the simple system of $\Psi$ with $\Gamma_{0}^{\prime} \subseteq \Phi_{+}$and define the corresponding coweights $\omega_{\alpha}^{\prime}:=\omega_{\alpha}\left(\Gamma_{0}^{\prime}\right)$ for $\alpha \in \Gamma_{0}^{\prime}$. Thus $\left\langle\alpha, \omega_{\beta}^{\prime}\right\rangle=\delta_{\alpha, \beta}$ if $\alpha, \beta \in \Gamma_{0}^{\prime}$. The group $X^{\prime}$ acts by translation on $P_{\mathbb{R}}(\Psi)=\mathbb{R} \Psi$. Since $X^{\prime}=\sum_{i: X_{i}^{\prime} \neq 0} \sum_{\alpha \in \Gamma_{0}^{\prime} \cap \Psi_{i}} \mathbb{Z} m_{i} k_{i, \alpha} \omega_{\alpha}^{\prime}$, it follows that the region

$$
D:=\left\{\sum_{\alpha \in \Gamma_{0}^{\prime}} e_{\alpha} \omega_{\alpha}^{\prime} \mid e_{\alpha} \in \mathbb{R}, 0 \leq e_{\alpha}<m_{i} k_{i, \alpha} \text { if } X_{i}^{\prime} \neq 0 \text { and } \alpha \in \Psi_{i}\right\} \subseteq \mathbb{R} \Psi
$$

is a fundamental domain for the action of $X^{\prime}$ by translation on $\mathbb{R} \Psi$. This gives a bijection $h: P_{\mathbb{R}}(\Psi) / X^{\prime} \cong D$ such that $h^{-1}(d)=d+X^{\prime}$ for all $d \in D$.

6.6. Let $\mathcal{B}$ denote the set of alcoves of $W^{\prime}$ acting on $\mathbb{R} \Psi$, i.e. the connected components of the complements of reflecting hyperplanes of $W^{\prime}$ in $\mathbb{R} \Psi$. Any alcove $B$ is a direct sum of simplices and simplicial cones lying in the pairwise orthogonal subspaces of $\mathbb{R} \Psi$ given by the linear spans of the components $\Psi_{i}$ of $\Psi$. Each alcove $B$ may be written as

$$
B=\left\{v \in \mathbb{R} \Psi \mid\langle\alpha, v\rangle>c_{\alpha} \text { for all } \alpha \in \Sigma\right\},
$$

where each $c_{\alpha} \in \mathbb{R}, \Sigma=\Sigma_{B} \subseteq \Psi$ and $\Sigma$ is minimal under inclusion. The hyperplanes $\left\{v \in \mathbb{R} \Psi \mid\langle\alpha, v\rangle=c_{\alpha}\right\}$ for $\alpha \in \Sigma_{B}$ are the walls of $B$. We define the lower closure $B_{0}$ of $B$ as

$$
\begin{aligned}
B_{0}=\left\{v \in \mathbb{R} \Psi \mid\langle\alpha, v\rangle \geq c_{\alpha} \text { for all } \alpha \in \Sigma \cap\right. & -\Phi_{+}, \\
& \left.\langle\alpha, v\rangle>c_{\alpha} \text { for all } \alpha \in \Sigma \cap \Phi_{+}\right\} .
\end{aligned}
$$

Thus $B_{0}$ is obtained by removing all points of some of the walls of $B$ from the topological closure $\bar{B}=\left\{v \in \mathbb{R} \Psi \mid\langle\alpha, v\rangle \geq c_{\alpha}\right.$ for all $\left.\alpha \in \Sigma\right\}$ of $B$. The walls removed are precisely those whose unit normal (outward from $B$ in $\mathbb{R} \Psi$ ) is a positive scalar multiple of a positive root (i.e. one in $\Psi \cap \Phi_{+}$).

6.7. Alcoves. Basic properties of alcoves and their lower closures are recorded in the following lemma.

Lemma 11. (a) $\mathbb{R} \Psi=\bigcup_{B \in \mathcal{B}} B_{0}$ (a disjoint union).

(b) If $B \in \mathcal{B}$ and $\lambda \in X^{\prime}$, then $C:=B+\lambda \in \mathcal{B}$ and $C_{0}=B_{0}+\lambda$.

(c) Every $v \in X^{\prime}$ is contained in $B_{0}$ for a unique $B \in \mathcal{B}$.

(d) Every $v \in Y^{\prime}$ is contained in $\bar{B}$ for a unique $B \in \mathcal{B}$.

(e) Let $B$ be an alcove for $W^{\prime}$ on $\mathbb{R} \Psi$ with $0 \in \bar{B}$. Let $G:=\{C \in \mathcal{B} \mid 0 \in \bar{C}\}$. Then $G=\left\{w B \mid w \in W_{\Psi}\right\}$. 
(f) The region $D^{\prime}=\dot{\bigcup}_{C \in G} C_{0}$ is a fundamental domain for the action on $\mathbb{R} \Psi$ of the translation subgroup $T=\left\{x \mapsto x+v: \mathbb{R} \Psi \rightarrow \mathbb{R} \Psi \mid v \in Y^{\prime}\right\}$ of $W^{\prime}$.

Proof. The notion of lower closure of an alcove is related in an obvious way to the notion of upper closure of a facet as discussed in [19, 6.1-6.2]. By simple modification of the discussion in [19, 6.2], one sees that (a)-(c) hold. For (d)-(e), see [2, Ch. V, §3, no. 10]. Finally, (f) follows from (a), (b), (e) and Proposition 1(a) applied to $W^{\prime}$.

6.8. By definition, $D^{\prime}$ is a union of lower closures of alcoves. We observe that the same holds for $D$.

Lemma 12. We have $D=\bigcup_{B \subseteq \mathcal{B}} B_{0}$.

Proof. Observe that

$$
D:=\left\{v \in \mathbb{R} \Psi \mid 0 \leq\langle v, \alpha\rangle<m_{i} k_{i, \alpha} \text { if } X_{i}^{\prime} \neq 0 \text { and } \alpha \in \Psi_{i} \cap \Gamma_{0}^{\prime}\right\} .
$$

It is easy to check that the walls of $D$ are reflecting hyperplanes of $W^{\prime}$. The desired conclusion then follows from Lemma 11(a) since the definition of lower closure and the form of the above inequalities defining $D$ imply that if $v \in D$ is in $B_{0}$ for some $B \in \mathcal{B}$, then $\mathcal{B}_{0} \subseteq D$.

6.9. Since $D^{\prime}$ is a fundamental domain for $Y^{\prime}$ acting by translation on $\mathbb{R} \Psi$, there is a unique map $k: D \rightarrow D^{\prime}$ such that $k(d)-d \in Y^{\prime}$ for all $d \in D$. For any alcove $B \in \mathcal{B}$ with $B \subseteq D$, there is a unique $\gamma_{B} \in Y^{\prime}$ such that $B_{0}+\gamma_{B} \subseteq D^{\prime}$. The map $k$ is determined by the condition that $k(d)=d+\gamma_{B}$ for all $B \in \mathcal{B}$ with $B \subseteq D$ and all $d \in B_{0}$. Obviously $k$ is injective, and the image of $k$ is the union of the lower closed alcoves in $D$ which are translates by elements of $Y^{\prime}$ of lower closed alcoves in $D$.

Remark 12. Let $B$ be some alcove of $W^{\prime}$ with $0 \in \bar{B}$. Consider the subgroup $G=\left\{w \in W_{\Psi} \mid w B=\tau_{\gamma} B\right.$ for some $\left.\gamma \in X^{\prime}\right\}$. It is well known (and follows from Lemma 11 and Proposition 11(a)) that $G \cong X^{\prime} / Y^{\prime}$, under the map taking each $w \in G$ to the coset $\gamma+Y^{\prime}$ where $\gamma \in X^{\prime}$ with $w B=\tau_{\gamma} B$. We have $\operatorname{Im}(k) \subseteq D^{\prime}=$ $\bigcup_{w \in W_{\psi}}(w B)_{0}$. Let $M:=\left\{w \in W_{\Psi} \mid(w B)_{0} \subseteq \operatorname{Im}(k)\right\}$. It is not difficult to see that $M$ is a set of coset representatives for $W^{\prime} / G$.

6.10. Recall that $\mathcal{R}_{\left(\Psi, X^{\prime}\right)}(\Phi, \mathbb{R}):=j_{\mathbb{R}}^{-1}\left(\mathcal{R}_{\left(\Psi, X^{\prime}\right)}^{\prime}(\Phi, \mathbb{R})\right)$. We shall require the following detailed description of this subset of $\mathcal{R}(\Phi, \mathbb{R})$.

Lemma 13. Let $(\Gamma, f) \in \mathcal{R}(\Phi, \mathbb{R})$. Then $(\Gamma, f) \in \mathcal{R}_{\left(\Psi, X^{\prime}\right)}(\Phi, \mathbb{R})$ if and only if the following conditions (i)-(iv) hold:

(i) There is some simple subsystem $\Gamma^{\prime}$ of $\Psi$ with $\Psi \supseteq \Gamma \supseteq \Gamma^{\prime}$.

(ii) If $X_{i}^{\prime}=0$, then $\Gamma \cap \Psi_{i}=\Gamma^{\prime} \cap \Psi_{i}$.

(iii) If $X_{i}^{\prime}=m_{i} P\left(\Psi_{i}\right)$ where $m_{i}>0$, then $\left(\Gamma \backslash \Gamma^{\prime}\right) \cap \Psi_{i}=\left\{\alpha_{i, 0}\right\}$ where $-\alpha_{i, 0}$ is the highest root of $\Psi_{i}$ with respect to its simple system $\Psi_{i} \cap \Gamma^{\prime}$, and $K_{i}=m_{i}$.

(iv) If $X_{i}^{\prime}=m_{i} P\left(\Psi_{i}^{0}\right)$ and $m_{i}>0$, then $\left(\Gamma \backslash \Gamma^{\prime}\right) \cap \Psi_{i}=\left\{\alpha_{i, 0}\right\}$ where $-\alpha_{i, 0}^{\vee}$ is the highest root of $\Psi_{i}^{\vee}$ with respect to its simple system $\left(\Psi_{i} \cap \Gamma^{\prime}\right)^{\vee}$, and $K_{i}=m_{i}$.

Here, if $X_{i}^{\prime} \neq 0$ and $\sum_{\alpha \in \Psi_{i} \cap \Gamma} c_{\alpha} \alpha=0$ with all $c_{\alpha} \in \mathbb{R}$ and $c_{\alpha_{i, 0}}=1$, we write $K_{i}:=\sum_{\alpha \in \Psi_{i} \cap \Gamma} c_{\alpha} f(\alpha)$. 
Proof. When $\mathbb{R}$ is replaced by $\mathbb{Z}$, this is implicit in the proof of Theorem 4 , see especially (20). The proof for $\mathbb{R}$ is essentially the same.

6.11. Let $\widehat{\mathcal{R}}_{\left(\Psi, X^{\prime}\right)}(\Phi, \mathbb{R})$ denote the set of all triples $x=\left(\Gamma, \Gamma^{\prime}, f\right)$ such that $(\Gamma, f) \in$ $\mathcal{R}(\Phi, \mathbb{R})$ and the conditions (i)-(iv) above hold. To $x=\left(\Gamma, \Gamma^{\prime}, f\right) \in \widehat{\mathcal{R}}_{\left(\Psi, X^{\prime}\right)}(\Phi, \mathbb{R})$, we attach a point $v_{x}:=\sum_{\alpha \in \Gamma^{\prime}} f(\alpha) \omega_{\alpha}\left(\Gamma^{\prime}\right) \in P_{\mathbb{R}}(\Psi)=\mathbb{R} \Psi$.

Lemma 14. Let $B$ be the (unique) alcove of $W^{\prime}$ in $\mathbb{R} \Psi$ such that $0 \in \bar{B}$ and $B$ is in the fundamental chamber for $W_{\Psi}$ with respect to its positive system $\Gamma^{\prime}$. Then:

(a) We have $j_{\mathbb{R}}(\Gamma, f)=\left(\Psi, v_{x}+X^{\prime}\right)$.

(b) The coset $v_{x}+X^{\prime} \in \mathbb{R} \Psi / X^{\prime}$ depends only on $(\Gamma, f)$ and not on $\Gamma^{\prime}$.

(c) For fixed $\left(\Gamma, \Gamma^{\prime}\right)$, the map $x=\left(\Gamma, \Gamma^{\prime}, f\right) \mapsto v_{x}$ defines a bijection from the set of triples $\left(\Gamma, \Gamma^{\prime}, f\right) \in \widehat{\mathcal{R}}_{\left(\Psi, X^{\prime}\right)}(\Phi, \mathbb{R})$ to the lower closure $B_{0}$ of $B$.

Proof. For (a), observe that $\vec{\Phi}^{a}(\Gamma, f)=\vec{\Phi}^{a}\left(\Psi, v_{x}+X^{\prime}\right)$ (cf. the proof of Theorem (4). Then (b) follows from (a). For (c), note that the only restrictions on the function $f$ are that $f(\alpha) \geq 0$ for all $\alpha \in \Gamma$, that $f(\alpha)>0$ if $\alpha \in-\Phi_{+} \cap \Gamma$, and that $\sum_{\alpha \in \Gamma_{i}} c_{\alpha} f(\alpha)=m_{i}$ if $\Gamma_{i}^{\prime} \neq \Gamma_{i}$. One checks, using this, that the set of points $v_{x}$ arising as in (b) is the set of all solutions $v$ of the following system of simultaneous inequalities for $v \in \mathbb{R} \Psi$ :

$$
\begin{cases}\langle v, \alpha\rangle \geq 0, & \text { for } \alpha \in \Gamma^{\prime} \cap \Psi_{+}, \\ \langle v, \alpha\rangle>0, & \text { for } \alpha \in \Gamma^{\prime} \cap-\Psi_{+}, \\ \langle v, \alpha\rangle \geq-m_{i}, & \text { for } \alpha \in\left(\Gamma \backslash \Gamma^{\prime}\right) \cap \Psi_{i,+}, \\ \langle v, \alpha\rangle>-m_{i}, & \text { for } \alpha \in\left(\Gamma \backslash \Gamma^{\prime}\right) \cap-\Psi_{i,+},\end{cases}
$$

where $\Psi_{+}=\Psi \cap \Phi_{+}$, etc. and $i$ in the last two inequalities is such that $\Gamma_{i} \neq \Gamma_{i}^{\prime}$. On the other hand, the alcove $B$ is defined by the set of inequalities above with each non-strict inequality replaced by strict inequality. The equations obtained by replacing inequalities with equalities define the walls of $B$. To see this, note that if $\alpha \in\left(\Gamma \backslash \Gamma^{\prime}\right) \cap \Psi_{i}$, then $k_{i, \alpha}=1$. From the definition of lower closure, the set of inequalities above also defines $B_{0}$.

6.12. There is a map $q_{k}: \widehat{\mathcal{R}}_{\left(\Psi, X^{\prime}\right)}(\Phi, k) \rightarrow \mathcal{R}_{\left(\Psi, X^{\prime}\right)}(\Phi, k)$ defined by $q\left(\Gamma, \Gamma^{\prime}, f\right)=$ $(\Gamma, f)$. Write $q=q_{\mathbb{Z}}$. Next define a function $g: D^{\prime} \rightarrow \widehat{\mathcal{R}}_{\left(\Psi, X^{\prime}\right)}(\Phi, \mathbb{R})$ as follows. Let $d \in D^{\prime}$. There is a unique alcove $B \in \mathcal{B}$ such that $0 \in \bar{B}$ and $d \in B_{0}$. Let $\Gamma^{\prime}$ be the unique simple system for $\Psi$ such that $B$ is contained in the fundamental chamber of $W_{\Psi}$ on $\mathbb{R} \Psi$ with respect to $\Gamma^{\prime}$. There is a unique subset $\Gamma$ of $\Psi$ such that $\left(\Gamma, \Gamma^{\prime}\right)$ satisfies the conditions of Lemma13)(i)-(iv) with the condition $K_{i}=m_{i}$ omitted in (iii)-(iv). By Lemma 14, there is a unique function $f: \Gamma \rightarrow \mathbb{R}$ such that $x:=\left(\Gamma, \Gamma^{\prime}, f\right) \in \mathcal{R}_{\left(\Psi, X^{\prime}\right)}(\Phi, \mathbb{R})$ and $v_{x}=d$. We define $g(d)=x$.

It is a simple consequence of Lemmas 11, 13 and 14 that the map $q$ is surjective and $g$ is a bijection.

6.13. We now come to the main result of this section.

Theorem 5. The bijection $\mathcal{R}_{\left(\Psi, X^{\prime}\right)}^{\prime}(\Phi, \mathbb{R}) \rightarrow \mathcal{R}_{\left(\Psi, X^{\prime}\right)}(\Phi, \mathbb{R})$ defined as the restriction to the left side of $j_{\mathbb{R}}^{-1}$ is given by $j_{\mathbb{R}}^{-1}(\Psi, X)=q g k h(X)$ for $X \in P_{\mathbb{R}}(\Psi) / X^{\prime}$,

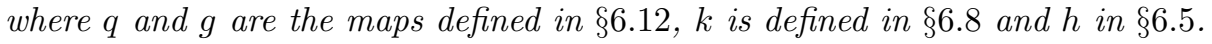


Proof. Write $h(X)=d$, so $X=d+X^{\prime}$. Set $g(d)=d^{\prime}$, so $d-d^{\prime} \in Y^{\prime} \subseteq X^{\prime}$ and $X=d^{\prime}+X^{\prime}$. Set $g\left(d^{\prime}\right)=\left(\Gamma, \Gamma^{\prime}, f\right)=x$. By definition of $g$, we have $v_{x}=d^{\prime}$ in Lemma 14. Then $j_{\mathbb{R}} q g k h(X)=j_{\mathbb{R}}(\Gamma, f)=\left(\Psi, v_{x}+X^{\prime}\right)=\left(\Psi, d^{\prime}+X^{\prime}\right)=(\Psi, X)$, and the theorem follows.

6.14. If $k=\mathbb{Z}$ and $X^{\prime}$ has finite index in $P(\Psi)$, then evidently

$$
\left|\mathcal{R}_{\left(\Psi, X^{\prime}\right)}^{\prime}(\Phi, \mathbb{Z})\right|=\left|\mathcal{R}_{\left(\Psi, X^{\prime}\right)}(\Phi, \mathbb{Z})\right| \in \mathbb{N} .
$$

This equality implies various identities in $\mathbb{Z}$ by counting the points of $\mathcal{R}_{\left(\Psi, X^{\prime}\right)}^{\prime}(\Phi, \mathbb{Z})$ and $\mathcal{R}_{\left(\Psi, X^{\prime}\right)}(\Phi, \mathbb{Z})$ according to the definitions. We shall formulate these identities explicitly below in the special case that $\Psi=\Phi$ is irreducible. In general, the identities easily reduce to those in this special case by considering indecomposable components of $\Psi$.

Assume that $\Psi=\Phi$ is indecomposable and $X^{\prime} \neq \emptyset$. We have either $X^{\prime}=M P(\Phi)$ or $X^{\prime}=M P\left(\Phi^{\circ}\right)$ for some $M \in \mathbb{N}_{>0}$, so $\left[P(\Phi): X^{\prime}\right]$ is finite. Fix the np subset $\Gamma:=\Pi \cup\{-\omega\}$ of $\Phi$, where $\omega$ is the highest root of $\Phi$ with respect to $\Pi$ if $X^{\prime}=P(\Phi)$ and $-\omega^{\vee}$ is the highest root of $\Psi^{\vee}$ with respect to $\Pi^{\vee}$ if $X^{\prime}=P\left(\Psi^{\circ}\right)$.

Lemma 15. Let $W$ act on np subsets $\Sigma \subseteq \Phi$ by $(w, \Sigma) \mapsto w(\Sigma)$. Then the setwise stabiliser of $\Gamma$ in $W$ satisfies $\operatorname{Stab}_{W}(\Gamma) \cong P(\Phi) / Q(\Phi)$ and in particular $\left|\operatorname{Stab}_{W}(\Gamma)\right|=f_{\Phi}$.

Proof. Suppose that $\omega$ is the highest root of $\Phi$ (the other case follows from this one by considering the np subset $\left.\Gamma^{\vee} \subseteq \Phi^{\vee}\right)$. Let $a: \widetilde{W}^{a} \rightarrow W$ denote the natural projection. Using the fact that $\pi(\widetilde{\Pi})=\Gamma$, it follows that $a$ restricts to a map $a^{\prime}: \operatorname{Stab}_{\widetilde{W}^{a}}(\widetilde{\Pi}) \rightarrow \operatorname{Stab}_{W}(\Gamma)$. Using the definition of $\widetilde{W}^{a}$, one readily checks that $a^{\prime}$ is surjective. The group $\operatorname{Stab}_{\widetilde{W}^{a}}(\widetilde{\Pi})=\operatorname{Stab}_{\widetilde{W}^{a}}(A)$ is discussed in detail (to the extent that its elements are explicitly described) in [2, Ch. VI, §2, no. 3]. From the results there, one has in particular that $\operatorname{Stab}_{\widetilde{W}^{a}}\left(\Pi^{\vee}\right) \cong P(\Phi) / Q(\Phi)$ and the restriction of $a$ to $\operatorname{Stab}_{\widetilde{W}^{a}}(\widetilde{\Pi})$ is injective. Hence

$$
\operatorname{Stab}_{W}(\Gamma)=a\left(\operatorname{Stab}_{\widetilde{W}^{a}}(\widetilde{\Pi})\right) \cong \operatorname{Stab}_{\widetilde{W}^{a}}(\widetilde{\Pi}) \cong P(\Phi) / Q(\Phi),
$$

and the result follows.

Remark 13. By considering irreducible components of $\Sigma$, one can determine (using the proof of the above lemma) the setwise stabiliser in $W_{\Sigma}$ of any np subset $\Sigma$ of $\Phi$. We do not go into the more delicate question of the stabiliser of $\Sigma$ in $W$.

6.15. Consider the integers $c_{\alpha} \in \mathbb{N}_{\geq 0}$ which are defined by $\sum_{\alpha \in \Gamma} c_{\alpha} \alpha=0$ and $c_{\alpha}=1$. Define a generalised "ordered partition function" $\wp^{(\Gamma)}: \mathbb{Z} \rightarrow \mathbb{N}$ by

$$
\begin{aligned}
\wp^{(\Gamma)}(M) & :=\left|\left\{f: \Gamma \rightarrow \mathbb{N} \mid \sum_{\alpha \in \Gamma} c_{\alpha} f(\alpha)=M\right\}\right| \\
& =\left|\left\{f: \Gamma^{\prime} \rightarrow \mathbb{N} \mid \sum_{\alpha \in \Gamma^{\prime}} c_{\alpha} f(\alpha) \leq M\right\}\right| .
\end{aligned}
$$

Define some non-standard descent statistics on $W$ as follows. For $w \in W$, define $d(w)=\left\{\alpha \in \Gamma \mid w(\alpha) \in \Phi_{-}\right\}$. For $i \in \mathbb{N}$, let $d_{i}:=\left|\left\{w \in W \mid \sum_{\alpha \in d(w)} c_{\alpha}=i\right\}\right|$. Set $h=\sum_{\alpha \in \Gamma} c_{\alpha}$ ( $h$ is the Coxeter number of $\Phi$ if $\omega$ is the highest root of $\Phi$, by [2, Ch. VI, §1, no. 11, Prop. 31]). Then $d_{i}=0$ if $i=0$ or $i \geq h$. 
Theorem 6. Let $N^{\prime}=\left|\Pi_{\text {long }}\right|$ and $N:=|\Pi|$. Then:

(a) For any $M \in \mathbb{N}_{>0}$,

$$
\sum_{i=0}^{h} \frac{d_{i}}{f_{\Phi}} \cdot \wp^{(\Gamma)}(M-i)= \begin{cases}M^{N}, & \text { if } \omega \in \Phi_{\text {long }}, \\ M^{N} k_{\Phi}^{N^{\prime}}, & \text { if } \omega \in \Phi_{\text {short }} .\end{cases}
$$

(b) We have $\frac{d_{i}}{f_{\Phi}} \in \mathbb{N}$ for all $i$.

Proof. For any $\Delta \subseteq \Gamma$, define $d_{\Delta}:=|\{w \in W \mid d(w)=\Delta\}|$. Note that $d_{\emptyset}=d_{\Gamma}=$ 0 . To prove formula (a), it will suffice to show that

$$
\frac{1}{f_{\Phi}} \sum_{\Delta \subseteq \Gamma} d_{\Delta} \cdot \wp^{(\Gamma)}\left(M-\sum_{\alpha \in \Delta} c_{\alpha}\right)= \begin{cases}M^{N}, & \text { if } \omega \in \Phi_{\text {long }} \\ M^{N} k_{\Phi}^{N^{\prime}}, & \text { if } \omega \in \Phi_{\text {short }}\end{cases}
$$

The right hand side is $|D \cap P(\Phi)|=\left|\mathcal{R}_{\left(\Psi, X^{\prime}\right)}^{\prime}(\Phi, \mathbb{Z})\right|$. On the other hand, consider the set $\widehat{\mathcal{R}}_{\left(\Psi, X^{\prime}\right)}(\Phi, \mathbb{Z})$. From Lemma 13 , one sees that a typical element of this set is of the form $\left(w(\Gamma), w\left(\Gamma^{\prime}\right), f \circ w^{-1}: w(\Gamma) \rightarrow \mathbb{N}\right)$ for (unique) $w \in W$ and $f: \Gamma \rightarrow \mathbb{N}$ such that $f(\alpha)>0$ if $w(\alpha) \in \Phi_{-}$and $\sum_{\alpha \in \Gamma} f(\alpha) c_{\alpha}=M$. For fixed $w$, the number of possible functions $f$ is therefore $\wp^{(\Gamma)}\left(M-\sum_{\alpha \in \Delta} c_{\alpha}\right)$, where $\Delta=\left\{\alpha \in \Gamma \mid w(\alpha) \in \Phi_{-}\right\}$. Hence the left hand side is equal to $\frac{1}{f_{\Phi}}\left|\widehat{\mathcal{R}}_{\left(\Psi, X^{\prime}\right)}(\Phi, \mathbb{Z})\right|$. But since $W$ acts simply transitively on the simple systems $\Gamma^{\prime}$ of $\Phi$, we get from Lemma 15 that each fiber of the map $q_{\mathbb{Z}}: \widehat{\mathcal{R}}_{\left(\Psi, X^{\prime}\right)}(\Phi, \mathbb{Z}) \rightarrow \mathcal{R}_{\left(\Psi, X^{\prime}\right)}(\Phi, \mathbb{Z})$ given by $\left(\Gamma, \Gamma^{\prime}, f\right) \mapsto(\Gamma, f)$ has cardinality $f_{\Phi}$, and so

$$
\left|\widehat{\mathcal{R}}_{\left(\Psi, X^{\prime}\right)}(\Phi, \mathbb{Z})\right|=f_{\Phi}\left|\mathcal{R}_{\left(\Psi, X^{\prime}\right)}(\Phi, \mathbb{Z})\right|=f_{\Phi}\left|\mathcal{R}_{\left(\Psi, X^{\prime}\right)}^{\prime}(\Phi, \mathbb{Z})\right| .
$$

This completes the proof of the formula.

It remains to show that $\frac{d_{i}}{f_{\Phi}} \in \mathbb{N}$ for all $i$. Let $G:=\operatorname{Stab}_{W}(\Gamma)$. One readily checks that for $w \in W, g \in G$ and $\alpha \in \Gamma$, we have $d(w g)=g^{-1} d(w)$ and $c_{\alpha}=c_{g(\alpha)}$. It follows readily from this that $d_{i}^{\prime}:=\left\{w \in W \mid \sum_{\alpha \in d(w)} c_{\alpha}=i\right\}$ is a union of left cosets of $G$ (i.e. satisfies $\left.d^{\prime}(i)=d^{\prime}(i) G\right)$, and so its order $d_{i}$ is divisible by $|G|=f_{\Phi}$.

Remark 14. (a) Using the longest element of $W$, one easily shows that the sequence $\left(d_{0}, d_{1}, \ldots, d_{h}\right)$ is symmetric, i.e. $d_{i}=d_{h-i}$ for $i=0, \ldots, h$. One might conjecture that this sequence is also strictly unimodal, i.e. $d_{0}<d_{1}<$ $\ldots<d_{k}$, where $k$ is the largest integer satisfying $2 k \leq h-1$.

(b) The formula in Theorem 6(a) is a polynomial relation in $M$ which holds for infinitely many values of $M$. It may therefore be regarded as an identity in which $M$ is an indeterminate.

6.16. We finish by interpreting the identity in Theorem 6(a) in case $\Phi$ is of type $A_{n}$, $n \geq 1$. Here $W$ is naturally identified as the symmetric group $S_{n+1}$ on $\{1, \ldots, n+1\}$. Consider the sequence $\left(i_{0}, i_{1}, i_{2}, \ldots, i_{n+1}\right):=(n+1,1,2, \ldots, n+1)$. For $\sigma \in W$, let

$$
d_{\sigma}=\left|\left\{k \in \mathbb{N} \mid 1 \leq k \leq n+1, \sigma\left(i_{k-1}\right)>\sigma\left(i_{k}\right)\right\}\right|
$$

denote the number of cyclically consecutive descents of $\sigma$. For $i=0, \ldots, n+1$, let $d_{i}:=\left|\left\{\sigma \in W \mid d_{\sigma}=i\right\}\right|$, so $d_{0}=d_{n+1}=0$, and by Theorem 6 (b) $n+1$ divides $d_{i}$ 
for each $i$. The identity of Theorem 6 then becomes

$$
M^{n}=\sum_{i=1}^{n} \frac{d_{i}}{n+1}\left(\begin{array}{c}
M+i-1 \\
n
\end{array}\right)
$$

where $M$ is an indeterminate.

This identity, which reflects the fact that the $d_{i}$ in the present case are the Eulerian numbers, is discussed in [26, §4.3d., p. 209]. Thus Theorem 6(a) generalises this relation between two bases of the space of polynomial functions.

\section{Appendix A. Comparison with the Results of Dynkin And others}

In this appendix, we give, for comparison, a brief treatment of results of Dynkin [11. Ch. $2, \S 5]$ on closed root subsystems of $\Phi$ along the lines of our results on root subsystems in general. We also mention connections between our results and those of other authors. Simple systems of closed root subsystems played an important role in [11, where they were called $\Pi$-systems and were characterised as follows:

Lemma 16. Let $\Phi$ be as above. Then $\Gamma \subseteq \Phi$ is a simple system of some closed root subsystem of $\Phi$ iff it satisfies the following conditions:

(i) $\Gamma$ is linearly independent,

(ii) for any $\alpha, \beta \in \Gamma, \alpha-\beta \notin \Phi$.

Proof. See [11, §2] or, more generally, [13, Proposition 2.2] and its preceding paragraph. A simple proof independent of the theory of Lie algebras can be given as follows. First, well known facts about root strings [2, Ch. VI, §1, no. 3] show that (ii) implies (ii) of Lemma 1, Hence if (i) and (ii) hold, then Lemma 1-implies that $\Gamma$ is a simple system of some root subsystem $\Psi$. To see that $\Psi$ is closed, one reduces to the case that $\Phi$ is of rank two using the fact that any two linearly independent roots in $\Psi$ are $W^{\prime}$-conjugate to roots in some rank two standard parabolic root subsystem of $\Psi$, where $W^{\prime}$ denotes the Weyl group of $\Psi$. On the other hand, a simple system $\Gamma$ of some closed subsystem satisfies (i) and (ii) (using the definition of closed subsystem and the fact that $\alpha-\beta \notin \Gamma$ for $\alpha, \beta \in \Gamma$ ).

See [12, 3.1] for further discussion of the above conditions. The following definition is equivalent to a definition which can be found in [11, Ch. 2, §5]; it is an analogue for closed root subsystems of Definition 2 ,

Definition 7. Let $\Psi_{1} \subsetneq \Psi_{2}$ be a root subsystem of $\Phi$, with $\Psi_{1}$ closed. We say that $\Psi_{2}$ is a closed elementary extension of $\Psi_{1}$ if there is a simple system $\Pi$ of $\Psi_{1}$ and an indecomposable component $\Sigma$ of $\Psi_{1}$ such that a simple system for $\Psi$ is obtained by deleting one element of $\Sigma$ from $\Gamma:=\Pi \cup\{-\theta\}$, where $\theta$ is a root of $\Sigma$ which lies in the chamber $\mathcal{C}$ defined by the positive system corresponding to $\Pi$ and if moreover $\Gamma$ satisfies condition (ii) in Lemma 16 .

Note that in this definition, $\Psi_{2}$ has a simple system satisfying (i)-(ii) of Lemma [16] so $\Psi_{2}$ is also a closed subsystem of $\Phi$. Also, $\theta$ must be the highest root of $\Sigma$. Otherwise, there would be some simple root $\alpha \in \Pi \cap \Sigma$ of $\Sigma$ such that $-\theta-\alpha \in \Sigma$, by properties of saturated sets of weights (see [17]) or the underlying representation theory. 
The following is equivalent to [11, Th. 5.2 and 5.3]:

Theorem 7. Let $\Phi$ be a finite crystallographic root system in the Euclidean space $V$, and let $\Psi \subsetneq \Phi$ be a closed root subsystem. Then there is a closed root subsystem $\widehat{\Psi}$ with $\Psi \subsetneq \widehat{\Psi} \subseteq \Phi$, such that either $\Psi$ is parabolic in $\widehat{\Psi}$ or $\widehat{\Psi}$ is a closed elementary extension of $\Psi$.

Proof. Let $\Gamma, \Psi_{+}, W^{\prime}$ and $\mathcal{C}$ be as defined in the first paragraph of the proof of Theorem 1. In addition, choose a vector space total ordering of $V$ such that $\Psi_{+}$is the set of positive elements of $\Psi$ in this ordering. Let $\alpha$ be the minimal element of $\Phi \backslash \Psi$ in this ordering. Then for any $\beta \in \Gamma$, we have $\alpha-\beta \notin \Phi$, since $\Psi$ is closed. In particular, $\langle\alpha, \beta\rangle \leq 0$ for all $\beta \in \Gamma$, so $\alpha \in-\mathcal{C}$. Note that since $\Psi$ is closed, Lemma 16 and the above shows that $\widetilde{\Gamma}:=\Gamma \cup\{\alpha\}$ satisfies (ii) of Lemma 16. In particular, any linearly independent subset of $\widetilde{\Gamma}$ is a simple system of some closed subsystem of $\Phi$. Now one proceeds exactly as in the part of the proof of Theorem 1 after the definition there of $\widetilde{\Gamma}$. The root subsystem $\widehat{\Psi}$ defined there has the properties required in the statement of the theorem to be proved here.

The proof above is essentially the same as in [11, Ch. 2, §5], except for Dynkin's more extensive use of the classification of finite Weyl groups (which can be avoided here using Lemma 3(b)). Making repeated use of Theorem 7 and keeping track only of isomorphism types, one obtains the following in the same way as we deduce Corollary 1 (the result is also a corollary of the results of Borel and de Siebenthal, as pointed out in [21]).

Corollary 7. Let $\Phi$ be a finite crystallographic root system in the Euclidean space $V$. The Dynkin diagram of any closed root subsystem of $\Phi$ is obtained by a sequence of operations of the following type on Dynkin diagrams, beginning with the Dynkin diagram of $\Phi$ :

(1) Delete any number of nodes.

(2) Replace some indecomposable component $\Sigma$ of the Dynkin diagram by the completed Dynkin diagram of $\Sigma$, and delete at least one node from the resulting affine component.

Corollaries 1 and 7 (resp., Theorems 11 and 7) enable the recursive determination up to isomorphism (resp., conjugacy) of the root subsystems and closed root subsystems of $\Phi$, and of the corresponding subgroups of $W$. We do not know any case-free description of the final results. Tables giving the explicit results for each type of root system and additional information about classification (in some cases, for only the maximal or maximal rank subsystems or subgroups) up to conjugacy, or automorphisms of $\Phi$ or $W$, can be found in [11] and [12]. The commentary [25] on the translated version [11] of [10] also discusses these tables.

\section{REFERENCES}

[1] A. Borel and J. De Siebenthal. Les sous-groupes fermés de rang maximum des groupes de Lie clos. Comment. Math. Helv., 23:200-221, 1949. MR0032659(11:326d) 
[2] N. Bourbaki. Éléments de mathématique. Fasc. XXXIV. Groupes et algèbres de Lie. Chapitre IV: Groupes de Coxeter et systèmes de Tits. Chapitre V: Groupes engendrés par des réflexions. Chapitre VI: systèmes de racines. Actualités Scientifiques et Industrielles, No. 1337. Hermann, Paris, 1968. MR0240238 (39:1590)

[3] R. W. Carter. Conjugacy classes in the Weyl group. Compositio Math., 25:1-59, 1972. MR0318337(47:6884)

[4] R. W. Carter. Lie algebras of finite and affine type, volume 96 of Cambridge Studies in Advanced Mathematics. Cambridge University Press, Cambridge, 2005. MR2188930 (2006i:17001)

[5] H. S. M. Coxeter. Finite groups generated by reflections and their subgroups generated by reflections. Proc. Camb. Phil. Soc., 30:466-482, 1934.

[6] M. J. Dyer. On rigidity of abstract root systems of Coxeter groups. preprint, 2007.

[7] M. J. Dyer and G. I. Lehrer. Real root subsystems of loop Kac-Moody root systems. preprint, 2009.

[8] Matthew Dyer. Reflection subgroups of Coxeter systems. J. Algebra, 135(1):57-73, 1990. MR.1076077 (91j:20100)

[9] Matthew J. Dyer and Cédric Bonnafé. Semidirect product decomposition of Coxeter groups. preprint, 2008.

[10] E. B. Dynkin. Semisimple subalgebras of semisimple Lie algebras. Mat. Sbornik N.S., 30(72):349-462 (3 plates), 1952. MR0047629 (13:904c)

[11] E. B. Dynkin. Semisimple subalgebras of semisimple Lie algebras. Amer. Math. Soc. Trans. (2), 6:111-244, 1957.

[12] A. A. Felikson and P. V. Tumarkin. Reflection subgroups of Euclidean reflection groups. Mat. Sb., 196(9):103-124, 2005. MR2195709 (2007c:20096)

[13] Anna Felikson, Alexander Retakh, and Pavel Tumarkin. Regular subalgebras of affine KacMoody algebras. J. Phys. A, 41(36):365204, 16 pp., 2008. MR2447864 (2009h:17025)

[14] Anna Felikson and Pavel Tumarkin. Euclidean simplices generating discrete reflection groups. European J. Combin., 28(4):1056-1067, 2007. MR2305573 (2008a:20067)

[15] Simon M. Goodwin. On generation of the root lattice by roots. Math. Proc. Cambridge Philos. Soc., 142(1):41-45, 2007. MR2296389 (2007m:17012)

[16] R. B. Howlett, P. J. Rowley, and D. E. Taylor. On outer automorphism groups of Coxeter groups. Manuscripta Math., 93(4):499-513, 1997. MR1465894 (98j:20056)

[17] James E. Humphreys. Introduction to Lie algebras and representation theory, volume 9 of Graduate Texts in Mathematics. Springer-Verlag, New York, 1978. MR499562 (81b:17007)

[18] James E. Humphreys. Reflection groups and Coxeter groups, volume 29 of Cambridge Studies in Advanced Mathematics. Cambridge University Press, Cambridge, 1990. MR1066460 (92h:20002)

[19] Jens Carsten Jantzen. Representations of algebraic groups, volume 107 of Mathematical Surveys and Monographs. American Mathematical Society, Providence, RI, 2003. MR2015057 (2004h:20061)

[20] Victor G. Kac. Infinite-dimensional Lie algebras. Cambridge University Press, Cambridge, 1990. MR1104219 (92k:17038)

[21] Richard Kane. Reflection groups and invariant theory. CMS Books in Mathematics/Ouvrages de Mathématiques de la SMC, 5. Springer-Verlag, New York, 2001. MR.1838580 (2002c:20061)

[22] Gustav Lehrer and Donald Taylor. Unitary reflection groups. Australian Mathematical Society Lecture Notes, 20. Cambridge University Press, Cambridge, 2009. MR2542964

[23] Gustav I. Lehrer. Rational points and Coxeter group actions on the cohomology of toric varieties. Ann. Inst. Fourier (Grenoble), 58(2):671-688, 2008. MR2410386 (2009c:14102)

[24] Robert V. Moody and Arturo Pianzola. Lie algebras with triangular decompositions. Canadian Mathematical Society Series of Monographs and Advanced Texts. John Wiley \& Sons Inc., New York, 1995. MR.1323858 (96d:17025)

[25] A. L. Onishchik. Comments on the paper "Semisimple subalgebras of semisimple Lie algebras". In Selected papers of E. B. Dynkin with commentary, pages 309-312. Amer. Math. Soc., Providence, RI; International Press, Cambridge, MA, 2000.

[26] Richard P. Stanley. Enumerative combinatorics. Vol. 1, volume 49 of Cambridge Studies in Advanced Mathematics. Cambridge University Press, Cambridge, 1997. With a foreword by Gian-Carlo Rota. Corrected reprint of the 1986 original. MR 1090542 (91m:05002) 
[27] Robert Steinberg. Lectures on Chevalley groups. Yale University, New Haven, Conn., 1968. Notes prepared by John Faulkner and Robert Wilson.

[28] John R. Stembridge. Some permutation representations of Weyl groups associated with the cohomology of toric varieties. Adv. Math., 106(2):244-301, 1994. MR.1279220 (95f:20011)

School of Mathematics and Statistics, University of Sydney, Sydney, 2006 Australia Current address: Department of Mathematics, 25 Hurley Building, University of Notre Dame, Notre Dame, Indiana 46556

School of Mathematics and Statistics, University of Sydney, Sydney, 2006 Australia 